\title{
CHEMISCHE BERICHTE
}

GEGRUNDET 1868

118. JAHRGANG

HERAUSGEGEBEN IM AUFTRAG DER

GESELLSCHAFT DEUTSCHER CHEMIKER

VON

K. HAFNER - W. KIRMSE - H. MUSSO - H. NÖTH • J. SAUER - E. WINTERFELDT

UNTER MITWIRKUNG VON

W. BECK - H. A. BRUNE - H. BUDZIKIEWICZ - W. LUUTTKE

REDAKTION: H. ZAHN

mit H. SCHILL, J. STREHLOW und A. WIELAND

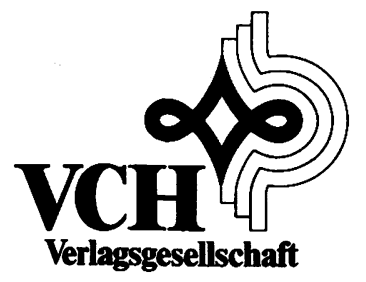




\section{INHALT VON HEFT 8}

\section{Jahrgang 1985}

\section{PHYSIKALISCHE CHEMIE}

Buschmann Hans-Jürgen: Komplexierung von Blei(II) durch Azakronenether und Kryptanden in Methanol ............................. 3408

\section{ANORGANISCHE CHEMIE}

Nöth Heinrich, Otto Peter und Storch Wolfgang: Beiträge zur Chemie des Bors, 155:

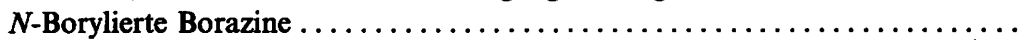
3020

Paul Wilfried und Werner Helmut: Basische Metalle, LVI: Reaktionen von (Pentamethylcyclopentadienyl)rhodium(I)-Komplexen mit Dihalogen- und Trihalo-

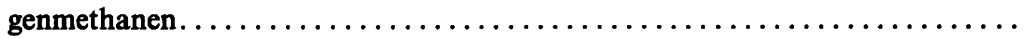

Schmidbaur Hubert, Herr Rudolf, Pollok Thomas, Schier Annette, Müller Gerhard und Riede Jürgen: Olefin- und Cyclopropan-Aktivierung durch geminale Phos-

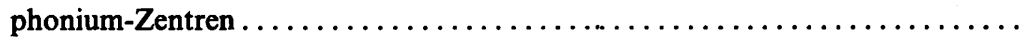

Sigwarth Beate, Weber Ute, Zsolnai Laszlo und Huttner Gottfried: Abfangreaktionen für Arsiniden- und Stibiniden-Komplexe: Addition von Lewisbasen an $\left[(\mathrm{CO})_{5} \mathbf{M}\right]_{2} \mathrm{XR} \quad(\mathrm{X}=\mathrm{As}, \mathbf{S b} ; \mathbf{M}=\mathbf{C r}, \mathbf{M o}, \mathbf{W}) \ldots \ldots \ldots \ldots \ldots \ldots \ldots \ldots \ldots$

Zeiher Christina, Hiller Wolfgang und Lorenz Ingo-Peter: Darstellung, Kristallstruktur und Eigenschaften von Diphenylphosphinito- $P$-metall( $(0)$-Komplexen des Typs $\left[\left(\mathrm{C}_{2} \mathrm{H}_{5}\right)_{3} \mathrm{NH}\right]\left[\mathrm{O}\left(\mathrm{C}_{6} \mathrm{H}_{5}\right)_{2} \mathrm{PM}(\mathrm{CO})_{5}\right](\mathrm{M}=\mathrm{Cr}, \mathrm{Mo}, \mathrm{W}) \ldots \ldots \ldots \ldots \ldots$

Beck Wolfgang, Bissinger Herbert, Castrillo de Castro Thais, Olgemöller Luitgard und Purucker Bernhard: Metallkomplexe mit biologisch wichtigen Liganden, XXXVII: Peptid-Synthese am Platin.........................

Herberhold Max, Ott Jutta und Haumaier Ludwig: Carbonylmetall-Komplexe mit Ferrocendithiocarboxylato-Liganden ........................

Schubert Ulrich, Heiser Bernd, Hee Lothar und Werner Helmut: Cycloadditionsreaktionen von Organometallkomplexen, VI: Ein Dimetalla-Spiroheterocyclus durch zweifache $[3+2]$-Cycloaddition einer Imidoylcobalt-Einheit und $\mathrm{CS}_{2} \ldots$

Arpac Ertugrul und Dahlenburg Lutz: Metallorganische Verbindungen des Iridiums und Rhodiums, XXIII: Chelatphosphan-substituierte Iridaheterocyclen des Typs

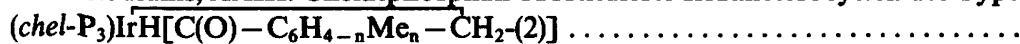

Paetzold Peter, Schröder Ernst, Schmid Günter und Boese Roland: (tert-Butylimino)[tert-butyl(trimethylsilyl)amino]boran, ein Aminoiminoboran, und seine

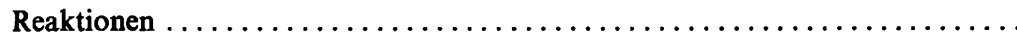

Niecke Edgar, Böske Jürgen, Krebs Bernt und Dartmann Mechthild: $\lambda^{5}$-Di- und -Triazaphospholene - Synthese, Isomerisierung und Zerfall .............

Haas Alois und Walz Rüdiger: Darstellung und chemisches Verhalten von S-(Trifluormethyl)-S,N-dihalogensulfimiden, (Trifluormethylthio)(thiosulfinyl)amin und Bis(trifluormethyldithio)schwefeldiimid $\ldots \ldots \ldots \ldots \ldots \ldots \ldots \ldots$

Wanjek Herbert, Nagel Ulrich und Beck Wolfgang: Metallkomplexe mit biologisch wichtigen Liganden, XXXVIII: Komplexe von Platin(II), Palladium(II), Nickel(II) und Kupfer(II) mit 1-Amino-1-cyclopropancarbonsäure-Derivaten . . 
Baudler Marianne und Göldner Wolfgang: Beiträge zur Chemie des Phosphors, 150: Aufbau eines tetracyclischen Phosphangerüstes aus einem Tricyclophosphan: Synthese der gemischt-substituierten Nonaphosphane(3) $\mathrm{P}_{9}(t \mathrm{Bu})_{2} \mathrm{X}(\mathrm{X}=\mathrm{Li}, \mathrm{H}$,

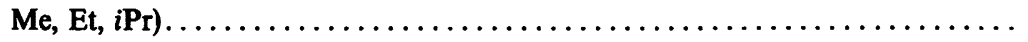

Fischer Ernst Otto, Wanner Jürgen Karl Roman, Müller Gerhard und Riede Jürgen: Übergangsmetall-Carben-Komplexe, CXXXVI: Reaktionen kationischer Carbin-Komplexe des Mangans mit Carbonylmetallaten.................

Hoffmann Gerhard G.: Komplexverbindungen von Trihalogeniden des Aluminiums und Galliums mit Thiolen, Schwefelwasserstoff und Selenolen ..............

Brunner Henri und Weber Hannelore: Asymmetrische Katalysen, 23: Optisch aktive Aminophosphane - Synthese und Verwendung in der Rh-katalysierten enantioselektiven Hydrosilylierung $\ldots \ldots \ldots \ldots \ldots \ldots \ldots \ldots \ldots \ldots \ldots \ldots$

Appel Rolf und Casser Carl: $[2+\mathrm{n}]$-Cycloadditionen des [Bis(trimethylsilyl)methylen $][($ trimethylsilyl)ethinyl $]$ phosphans $\ldots \ldots \ldots \ldots \ldots \ldots \ldots \ldots$

\section{ORGANISCHE CHEMIE}

Lerche Holger, Fischer Hans und Severin Theodor: Versuche zur Darstellung von

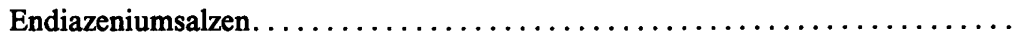

Griesbaum Karl und Zwick Gerhard: Monoozonolysen von acyclischen konjugierten

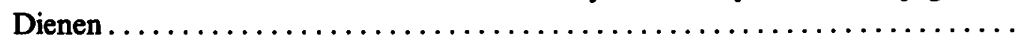

Quast Helmut, Eckert Philipp, Seiferling Bernhard, Peters Eva-Maria, Peters Karl und Schnering von Hans Georg: Photolyse des 9-Azidotriptycens. - Struktur des Dimeren eines extrem gespannten Brückenkopfimins . . . . . . . . . .

Adam Waldemar, De Lucchi Ottorino, Hill Karlheinz, Peters Eva-Maria, Peters Karl und Schnering von Hans Georg: Synthese, Thermolyse und Photolyse der Azoalkane Spiro[4,5-diazatricyclo[4.3.0.0.7.7 $]$ non-4-en-8,2'-[1,3]dioxolan] und 4,5Diazatricyclo[4.3.0.0 $0^{3,7}$ ]non-4-en-8-on. Über den Mechanismus der Oxadi- $\pi$-methan-Umlagerung von 5-Norbornen-2-on. . . . . . . . . . . . . . . .

Kupfer Rainer, Nagel Michael, Würthwein Ernst-Ulrich und Allmann Rudolf: Synthese und Struktur von $N$-Acylimidsäureestern ...................

Beck Wolfgang, Bissinger Herbert, Castrillo de Castro Thais, Olgemöller Luitgard und Purucker Bernhard: Metallkomplexe mit biologisch wichtigen Liganden, XXXVII: Peptid-Synthese am Platin .........................

Stahl Ingfried: 1,3-Dithienium- und 1,3-Dithioleniumsalze, III: Synthese cyclischer Dithioacetale von $\beta$-Ketoestern aus Keten-silylacetalen. . . . . . . . . . . . .

Stahl Ingfried: 1,3-Dithienium- und 1,3-Dithioleniumsalze, IV: 1,3-Dithian-2-yliumtetrafluoroborate - neue Agenzien zur Synthese von 1-Deuterioaldehyden....

Stetter Hermann und Simons Leo: Addition von Aldehyden an aktivierte Doppelbindungen, XXXVI: Synthesen und Reaktionen von 3-Acyl-1,5-dicarbonyl-

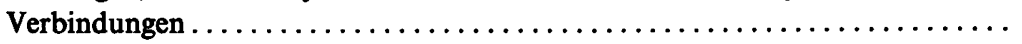

Maier Günther, Franz Lothar Hermann, Hartan Hans-Georg, Lanz Klaus und Reisenauer Hans Peter: Kleine Ringe, 54: Cyclopentadienon .............

Neidlein Richard und Klotz Udo J.: Synthesen substituierter elektronenreicher $N$-Sulfonyl-1-azabutadiene $\ldots \ldots \ldots \ldots \ldots \ldots \ldots \ldots \ldots \ldots \ldots \ldots \ldots \ldots \ldots \ldots \ldots \ldots \ldots$ 
Goerdeler Joachim, Linden Hans Werner, Puff Heinrich und Hundt Rudolf: Zur Kenntnis der Cycloaddukte aus 3-Imino-3H-1,2,4-dithiazolen und Nitrilen: Herstellungsbedingungen und Molekülstruktur . . . . . . . . . . . . . .

Wanjek Herbert, Nagel Ulrich und Beck Wolfgang: Metallkomplexe mit biologisch wichtigen Liganden, XXXVIII: Komplexe von Platin(II), Palladium(II), Nickel(II) und Kupfer(II) mit 1-Amino-1-cyclopropancarbonsäure-Derivaten . .

Nonnenmacher Axel, Plieningert Hans und Ziegler Manfred L.: Hochdruckversuche, XIII: Die Reaktion von tert-Butylisonitril und von Toluol mit Bicyclo[2.2.1]hepten unter Hochdruckbedingungen.$\ldots \ldots \ldots \ldots \ldots \ldots \ldots \ldots \ldots$

Jessen Jörg Lorenz, Schröder Gunter und Tochtermann Werner: Synthese mittlerer und großer Ringe, XI: Bicyclo[n.2.2]alkene aus überbrückten Oxepinen - Synthese von 3,6-Pentanooxepin-4,5-dicarbonsäure-dimethylester und 6,9-Dihydro[5]paracyclophan-7,8-dicarbonsäure-dimethylester.............

Noe Christian R., Knollmüller Max, Wagner Ernst und Völlenkle Horst: Kohlenhydrat-Modelle, I: Kinetische und thermodynamische Effekte bei Acetalisierungsreaktionen enantiomerenreiner Thiolactole $\ldots \ldots \ldots \ldots \ldots \ldots \ldots \ldots \ldots$

Sucrow Wolfgang, Minas Hermann, Stegemeyer Horst, Geschwinder Peter, Murawski Hans-Rüdiger und Krüger Carl: Aliphatische Flüssigkristalle, 2: Einige nematische Derivate des all-trans-Perhydrophenanthrens $\ldots \ldots \ldots \ldots \ldots \ldots \ldots$

Sucrow Wolfgang und Wolter Herbert: Aliphatische Flüssigkristalle, 3: Flüssig-kristalline 2-Cyclohexyldecaline $\ldots \ldots \ldots \ldots \ldots \ldots \ldots \ldots \ldots \ldots \ldots$

Adam Waldemar, Gretzke Nanette, Hasemann Ludwig, Klug Günter, Peters EvaMaria, Peters Karl, Schnering von Hans Georg und Will Bernd: Cycloaddition von Singulettsauerstoff und 4-Phenyl-4H-1,2,4-triazol-3,5-dion mit 7-substituier-

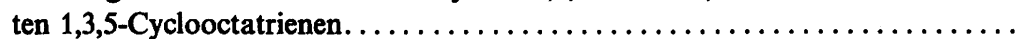

Theis Wolfgang und Regitz Manfred: Untersuchungen an Diazoverbindungen und Aziden, LX: Reaktionen von 3H-1,2,4-Triazol-3,5(4H)-dionen mit Vinyldiazo-

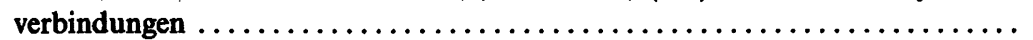

Bayer Heiner, Schreyer Michael, Gieren Alfred und Lamm Viktor: Decarboxylierungsprodukt von Hexahydrophthalsäureanhydrid: Struktur und spektrosko-

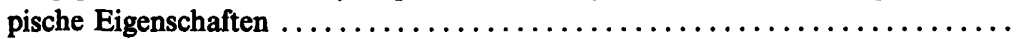

Reichardt Christian und Kaufmann Norbert: Eine verbesserte Methode zur Darstellung von 1,3-Dimethyl- und 1,3-Diethyl-2-methylenbenzimidazolin......... 


\section{PHYSICAL CHEMISTRY}

Buschmann Hans-Jürgen: Complexation of Lead(II) by Aza Crown Ethers and Cryp-

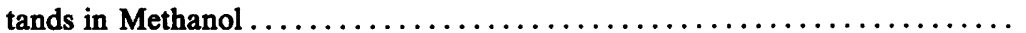

\section{INORGANIC CHEMISTRY}

Nöth Heinrich, Otto Peter, and Storch Wolfgang: Contributions to the Chemistry of

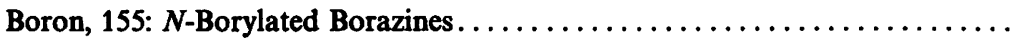

Paul Wilfried and Werner Helmut: Basic Metals, LVI: Reactions of (Pentamethylcyclopentadienyl)rhodium(I) Complexes with Dihalo- and Trihalomethanes ...

3032

Schmidbaur Hubert, Herr Rudolf, Pollok Thomas, Schier Annette, Müller Gerhard, and Riede Jürgen: Olefin and Cyclopropane Activation Through Geminal Phos-

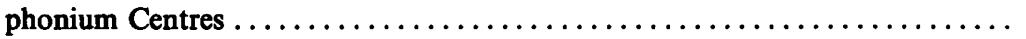

Sigwarth Beate, Weber Ute, Zsolnai Laszlo, and Huttner Gottfried: Trapping of Arsinidene and Stibinidene Intermediates: Addition of Lewis Bases to $\left[(\mathrm{CO})_{5} \mathbf{M}\right]_{2} \mathrm{XR}(\mathrm{X}=\mathrm{As}, \mathrm{Sb} ; \mathbf{M}=\mathbf{C r}, \mathbf{M o}, \mathrm{W}) \ldots \ldots \ldots \ldots \ldots \ldots \ldots \ldots \ldots \ldots$

Zeiher Christina, Hiller Wolfgang, and Lorenz Ingo-Peter: Synthesis, Crystal Structure, and Properties of Diphenylphosphinito-P-Transition Metal(0) Complexes of the Type $\left[\left(\mathrm{C}_{2} \mathrm{H}_{5}\right)_{3} \mathrm{NH}\right]\left[\mathrm{O}\left(\mathrm{C}_{6} \mathrm{H}_{5}\right)_{2} \mathrm{PM}(\mathrm{CO})_{5}\right](\mathrm{M}=\mathrm{Cr}$, Mo, W $) \ldots \ldots \ldots$

Beck Wolfgang, Bissinger Herbert, Castrillo de Castro Thais, Olgemöller Luitgard, and Purucker Bernhard: Metal Complexes with Biologically Important Ligands, XXXVII: Peptide Synthesis at Platinum(II) Ions. .................

Herberhold Max, Ott Jutta, and Haumaier Ludwig: Carbonylmetal Complexes with Ferrocenedithiocarboxylato Ligands. .......................

Schubert Ulrich, Heiser Bernd, Hee Lothar, and Werner Helmut: Cycloaddition Reactions of Organometal Complexes, VI: Synthesis of a Dimetalla Spiroheterocycle by Twofold $[3+2]$-Cycloaddition of an Imidoylcobalt Unit and $\mathrm{CS}_{2} \ldots$

Arpac Ertugrul and Dahlenburg Lutz: Organometallic Compounds of Iridium and Rhodium, XXIII: Chelate Phosphane-Substituted Iridaheterocycles of the Form (chel- $\left.\mathrm{P}_{3}\right) \mathrm{IrH}\left[\mathrm{C}(\mathrm{O})-\mathrm{C}_{6} \mathrm{H}_{4-\mathrm{n}} \mathrm{Me}_{\mathrm{n}}-\mathrm{CH}_{2}-(2)\right] \ldots \ldots \ldots \ldots \ldots \ldots \ldots \ldots \ldots \ldots \ldots \ldots$

Paetzold Peter, Schröder Ernst, Schmid Günter, and Boese Roland: (tert-Butylimi= no)[tert-butyl(trimethylsilyl)amino]borane, an Aminoiminoborane, and its

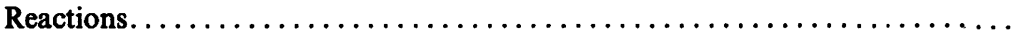

Niecke Edgar, Böske Jürgen, Krebs Bernt, and Dartmann Mechthild: $\lambda^{5}$-Di- and -Triazaphospholines - Synthesis, Isomerization, and Decomposition .......

Haas Alois and Walz Rüdiger: Preparation and Chemical Properties of S-(Trifluoromethyl)-S,N-dihalosulfimides, (Trifluoromethylthio)(thiosulfinyl)amine, and Bis(trifluoromethyldithio)sulfur Diimide......................

Wanjek Herbert, Nagel Ulrich, and Beck Wolfgang: Metal Complexes with Biologically Important Ligands, XXXVIII: Complexes of Platinum(II), Palladium(II), Nickel(II), and Copper(II) with 1-Amino-1-cyclopropanecarboxylic Acid Derivatives 
Baudler Marianne and Göldner Wolfgang: Contributions to the Chemistry of Phosphorus, 150: Synthesis of a Tetracyclophosphane Skeleton from a Tricyclophosphane: Synthesis of the Mixed-substituted Nonaphosphanes(3) $\mathbf{P}_{9}(t \mathrm{Bu})_{2} \mathrm{X}$

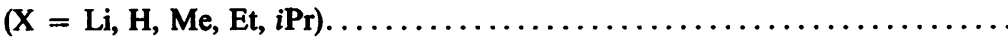

Fischer Ernst Otto, Wanner Jürgen Karl Roman, Müller Gerhard, and Riede Jürgen: Transition Metal Carbene Complexes, CXXXVI: Reactions of Cationic Carbyne Complexes of Manganese with Carbonyl Metalates..................

Hoffmann Gerhard G.: Complexes from the Trihalides of Aluminium and Gallium with Thiols, Hydrogen Sulfide, and Selenols ......................

Brunner Henri and Weber Hannelore: Asymmetric Catalyses, 23: Optically Active Aminophosphanes - Synthesis and Use in the Rh-Catalyzed Enantioselective

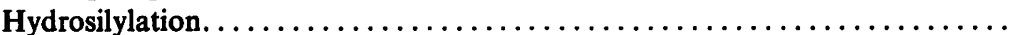

Appel Rolf and Casser Carl: [2+ n]-Cycloadditions of [Bis(trimethylsilyl)methylene]$[($ trimethylsilyl)ethynyl $]$ phosphane $\ldots \ldots \ldots \ldots \ldots \ldots \ldots \ldots \ldots \ldots \ldots$

\section{ORGANIC CHEMISTRY}

Lerche Holger, Fischer Hans, and Severin Theodor: Experiments to Form Enediazenium Salts . . . . . . . . . . . . . . . . . . . . . . . . . . . . .

Griesbaum Karl and Zwick Gerhard: Monoozonolyses of Acyclic Conjugated

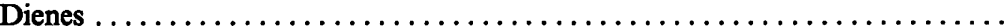

Quast Helmut, Eckert Philipp, Seiferling Bernhard, Peters Eva-Maria, Peters Karl, and Schnering von Hans Georg: Photolysis of 9-Azidotriptycene. - Structure of the Dimer of a Severely Strained Bridgehead Imine $\ldots \ldots \ldots \ldots \ldots \ldots \ldots$

Adam Waldemar, De Lucchi Ottorino, Hill Karlheinz, Peters Eva-Maria, Peters Karl, and Schnering von Hans Georg: Synthesis, Thermolysis and Photolysis of the Azoalkanes Spiro[4,5-diazatricyclo[4.3.0.0 $\left.0^{3,7}\right]$ non-4-ene-8, $2^{\prime}-[1,3]$ dioxolane $]$ and 4,5-Diazatricyclo[4.3.0.0 $\left.0^{3,7}\right]$ non-4-en-8-one. On the Mechanism of the Oxadi- $\pi$-methane Rearrangement of 5-Norbornen-2-one..............

Kupfer Rainer, Nagel Michael, Würthwein Ernst-Ulrich, and Allmann Rudolf: Syn-

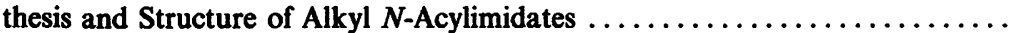

Beck Wolfgang, Bissinger Herbert, Castrillo de Castro Thais, Olgemöller Luitgard, and Purucker Bernhard: Metal Complexes with Biologically Important Ligands, XXXVII: Peptide Synthesis at Platinum(II) Ions. ..................

Stahl Ingfried: 1,3-Dithienium- and 1,3-Dithiolenium Salts, III: Synthesis of Cyclic Dithioacetals of $\beta$-Keto Esters from Ketene Silyl Acetals . . . . . . . . . . . . . .

Stahl Ingfried: 1,3-Dithienium- and 1,3-Dithiolenium Salts, IV: 1,3-Dithian-2-ylium Tetrafluoroborates - New Agents for the Synthesis of 1-Deuterioaldehydes...

Stetter Hermann and Simons Leo: Addition of Aldehydes to Activated Double Bonds, XXXVI: Syntheses and Reactions of 3-Acyl-1,5-dicarbonyl Compounds.......

Maier Günther, Franz Lothar Hermann, Hartan Hans-Georg, Lanz Klaus, and Reisenauer Hans Peter: Small Rings, 54: Cyclopentadienone .............

Neidlein Richard and Klotz Udo J.: Syntheses of Substituted Electron-rich $\mathrm{N}$-Sul-

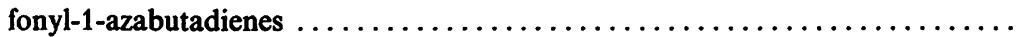


Goerdeler Joachim, Linden Hans Werner, Puff Heinrich, and Hundt Rudolf: Cycloadducts from 3-Imino-3H-1,2,4-dithiazoles and Nitriles: Conditions of Preparation and Structure

Wanjek Herbert, Nagel Ulrich, and Beck Wolfgang: Metal Complexes with Biologically Important Ligands, XXXVIII: Complexes of Platinum(II), Palladium(II), Nickel(II), and Copper(II) with 1-Amino-1-cyclopropanecarboxylic Acid Derivatives

Nonnenmacher Axel, Plieninger † Hans, and Ziegler Manfred L.: High Pressure Experiments, XIII: Reaction of tert-Butyl Isonitrile and of Toluene with Bicyclo[2.2.1] heptene (Norbornene) at High Pressure .................

Jessen Jörg Lorenz, Schröder Gunter, and Tochtermann Werner: Synthesis of Medium and Large Rings, XI: Bicyclo[n.2.2]alkenes from Bridged Oxepins - Synthesis of Dimethyl 3,6-Pentanooxepin-4,5-dicarboxylate and Dimethyl 6,9-Dihydro[5]paracyclophane-7,8-dicarboxylate $\ldots \ldots \ldots \ldots \ldots \ldots \ldots \ldots \ldots$

Noe Christian R., Knollmüller Max, Wagner Ernst, and Völlenkle Horst: Carbohydrate-Model Compounds, I: Kinetic and Thermodynamic Effects in Acetal Formation of Enantiomerically Pure Thiolactols

Sucrow Wolfgang, Minas Hermann, Stegemeyer Horst, Geschwinder Peter, Murawski Hans-Rüdiger, and Krüger Carl: Aliphatic Liquid Crystals, 2: Some Nematic Derivatives of all-trans-Perhydrophenanthrene ....................

Sucrow Wolfgang and Wolter Herbert: Aliphatic Liquid Crystals, 3: Liquid Crystalline 2-Cyclohexyldecalines. . . . . . . . . . . . . . . . . . . . . . . .

Adam Waldemar, Gretzke Nanette, Hasemann Ludwig, Klug Günter, Peters EvaMaria, Peters Karl, Schnering von Hans Georg, and Will Bernd: Cycloaddition of Singlet Oxygen and 4-Phenyl-4H-1,2,4-triazole-3,5-dione to 7-Substituted

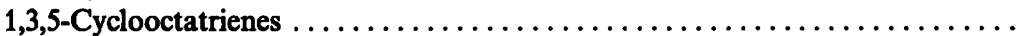

Theis Wolfgang and Regitz Manfred: Investigations on Diazo Compounds and Azides, LX: Reactions of $3 H-1,2,4-T r i a z o l e-3,5(4 H)$-diones with Vinyl Diazo

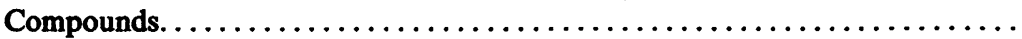

Bayer Heiner, Schreyer Michael, Gieren Alfred, and Lamm Viktor: Product of Decarboxylation of Hexahydrophthalic Anhydride: Structure and Spectroscopic Properties

Reichardt Christian and Kaufmann Norbert: An Improved Method for the Synthesis of 1,3-Dimethyl- and 1,3-Diethyl-2-methylenebenzimidazoline 
Adam, W., De Lucchi, O., Hill, K., Peters, E.-M., Peters, $K$. und Schnering, von, H. G. . . . . 3070

-, Gretzke, N.,. Hasemann, L.,

Klug, G., Peters, E.-M., Peters, K.,

Schnering, von, H. G. und Will, B. 3357

Allmann, R. s. Kupfer, B. ........ 3089

Appel, R. und Casser, C. ........ 3419

Arpac, E. und Dahlenburg, L. .... 3188

Baudler, M. und Göldner, W. ..... 3268

Bayer, $\boldsymbol{H}$., Schreyer, $M$.,

Gieren, A. und Lamm, $V$. 3413

Beck, W., Bissinger, $H$., Castrillo de

Castro, $T$., Olgemöller, $L$.

und Purucker, $B$.

- s. Wanjek, $\boldsymbol{H}$. 3258

Bissinger, H. s. Beck, W. . . . ... 3135

Boese, R. s. Paetzold, P. . . . . . . 3205

Böske, J. s. Niecke, E. . . . . . . . . 3227

Brunner, H. und Weber, H. ...... 3380

Buschmann, H.-J. . . . . . . . . 3408

Casser, C. s. Appel, R. ......... 3419

Castrillo de Castro, T. s. Beck, W. . . 3135

Dahlenburg, L. s. Arpac, E. ...... 3188

Dartmann, M. s. Niecke, E. . . . . . . 3227

De Lucchi, O. s. Adam, W. . . . . . 3070

Eckert, P. s. Quast, H. . . . . . . . . 3058

Fischer, E. O., Wanner, J. K. R.,

Müller, G. und Riede, J. . . . . 3311

Fischer, H. s. Lerche, H. . . . . . 3011

Franz, L. H. s. Maier, G. . . . . . . 3196

Geschwinder, P. s. Sucrow, W. . ... 3332

Gieren, A. s. Bayer, H. . . . . . . . 3413

Göldner, W. s. Baudler, M. ...... 3268

Goerdeler, J., Linden, $H$. $W$.,

Puff, $H$. und Hundt, $R$. ...... 3241

Gretzke, N. s. Adam, W. ........ 3357

Griesbaum, K. und Zwick, G. ...... 3041

Haas, A. und Walz, R. ........ 3248

Hartan, H.-G. s. Maier, G. . . . . . 3196
Hasemann, L. s. Adam, W. . . . . . 3357

Haumaier, L. s. Herberhold, M. . ... 3143

Hee, L. s. Schubert, U. . . . . . . 3151

Heiser, B. s. Schubert, U. . . . . . 3151

Herberhold, M., Ott, J. und

Haumaier, L. ............. 3143

Herr, R. s. Schmidbaur, H. ...... 3105

Hill, K. s. Adam, W. ........... 3070

Hiller, $W$. s. Zeiher, C. ........ 3127

Hoffmann, G. G. . .......... 3320

Hundt, $R$. s. Goerdeler, J. . . . . . 3241

Huttner, G. s. Sigwarth, B. . . . . . . 3114

Jessen, J. L., Schröder, G. und

Tochtermann, W. .......... 3287

Kaufmann, N. s. Reichardt, C. .... 3424

Klotz, U. J. s. Neidlein, R. . . . . ... 3217

Klug, G. s. Adam, W. . ......... 3357

Knollmüller, M. s. Noe, C. R. . . ... 3299

Krebs, B. s. Niecke, E. . . . . . . . 3227

Krüger, C. s. Sucrow, $W . \ldots \ldots \ldots 3332$

Kupfer, R., Nagel, M.,

Würthwein, $E .-U$. und

Allmann, R. .............. 3089

Lamm, $V$. s. Bayer, H. . . . . . . . . 3413

Lanz, K. s. Maier, G. . . ......... 3196

Lerche, $H$., Fischer, $H$. und

Severin, $T$. ............ 3011

Linden, $\boldsymbol{H}$. W. s. Goerdeler, J. . . . . 3241

Lorenz, I.-P. s. Zeiher, C. . ...... 3127

Maier, G., Franz, L. H.,

Hartan, H.-G., Lanz, K. und

Reisenauer, H. P. ......... 3196

Minas, $H$. s. Sucrow, $W . \ldots \ldots \ldots 3332$

Müller, G. s. Fischer, E. O. ...... 3311

- s. Schmidbaur, H. .......... 3105

Murawski, H.-R. s. Sucrow, W. . . . . 3332

Nagel, M. s. Kupfer, R. . ........ 3089

Nagel, $U$. s. Wanjek, H. ........ 3258

Neidlein, R. und Klotz, U. J. ...... 3217 
Niecke, E., Böske, J., Krebs, B.

und Dartmann, $M$. 3227

Noe, C. R., Knollmüller, M.,

Wagner, E. und Völlenkle, H. . . 3299

Nöth, H., Otto, P. und

Storch, $W$. 3020

Nonnenmacher, A., Plieninger $t, H$.

und Ziegler, M. L. ........ 3275

Olgemöller, L. s. Beck, W. . . . . . 3135

Ott, J. s. Herberhold, M. ........ 3143

Otto, P. s. Nöth, H. . ........... 3020

Paetzold, P., Schröder, E., Schmid, G.

und Boese, $R$. ............ 3205

Paul, W. und Werner, H. ...... 3032

Peters, E.-M. s. Adam, W. . . . 3070, 3357

- s. Quast, H. ............. 3058

Peters, K. s. Adam, W. . . . . 3070, 3357

- s. Quast, H. .............. 3058

Plieningert, $\boldsymbol{H}$. s. Nonnenmacher, A. 3275

Pollok, T. s. Schmidbaur, H. . . . ... 3105

Puff, H. s. Goerdeler, J. . . . . . . 3241

Purucker, B. s. Beck, W. ........ 3135

Quast, H., Eckert, P., Seiferling, B.,

Peters, E.-M., Peters, K. und

Schnering, von, H. G. . ...... 3058

Regitz, M. s. Theis, W. . . . . . . 3396

Reichardt, C. und Kaufmann, N. ... 3424

Reisenauer, H. P. s. Maier, G. .... 3196

Riede, J. s. Fischer, E. O. . . . . . . 3311

- s. Schmidbaur, H. .......... 3105

Schier, A. s. Schmidbaur, H. ...... 3105

Schmid, G. s. Paetzold, P. ....... 3205

Schmidbaur, H., Herr, R., Pollok, T.,

Schier, A., Müller, G. und

Riede, J. . ............. 3105

Schnering, von, $H$. G. s.

Adam, $W$.

3070,3357

- s. Quast, $H$.

3058
Schreyer, M. s. Bayer, H. ...... 3413

Schröder, E. s. Paetzold, P. ....... 3205

Schröder, G. s. Jessen, J. L. . . . . . 3287

Schubert, $U$., Heiser, B.,

Hee, L. und Werner, H. ...... 3151

Severin, T. s. Lerche, H. . ....... 3011

Seiferling, B. s. Quast, $H . \ldots \ldots \ldots 3058$

Sigwarth, B., Weber, $U$.,

Zsolnai, L. und Huttner, G. . ... 3114

Simons, L. s. Stetter, H. . . . . . . 3172

Stahl, I. .............. 3159, 3166

Stegemeyer, H. s. Sucrow, W. . . . . 3332

Stetter, H. und Simons, $L . \ldots \ldots \ldots 3172$

Storch, W. s. Nöth, H. . ........ 3020

Sucrow, W., Minas, H., Stegemeyer, $H$.,

Geschwinder, P., Murawski, H.-R.

und Krüger, C. ........... 3332

- und Wolter, H. ............. 3350

Theis, W. und Regitz, M. ....... 3396

Tochtermann, W. s. Jessen, J. L. . . . 3287

Völlenkle, H. s. Noe, C. R. . ...... 3299

Wagner, E. s. Noe, C. R. . ....... 3299

Walz, R. s. Haas, A. .......... 3248

Wanjek, $H$., Nagel, U. und Beck, W. 3258

Wanner, J. K. R. s. Fischer, E. O. ... 3311

Weber, H. s. Brunner, H. . . ..... 3380

Weber, $U$. s. Sigwarth, B. . ...... 3114

Werner, H. s. Paul, W. . . . . . . . 3032

- s. Schubert, U. ............. 3151

Will, B. s. Adam, W. . ........ 3357

Wolter, H. s. Sucrow, W. . . . . . 3350

Würthwein, E.-U. s. Kupfer, R. . . . . 3089

Zeiher, C., Hiller, W. und Lorenz, I.-P.

3127

Ziegler, M. L. s. Nonnenmacher, A. . . 3275

Zsolnai, L. s. Sigwarth, B. . . . . . 3114

Zwick, G. s. Griesbaum, K. ....... 3041 
Chem. Ber. 118, 3299-3310 (1985)

Kohlenhydrat-Modelle, I

\title{
Kinetische und thermodynamische Effekte bei Acetalisierungsreaktionen enantiomerenreiner Thiolactole
}

\author{
Christian R. Noe*a, Max Knollmüller ${ }^{\mathrm{a}}$, Ernst Wagner $^{\mathrm{a}}$ \\ und Horst Völlenkle
}

Institut für Organische Chemie der Technischen Universität Wien ${ }^{\mathrm{a}}$, Getreidemarkt 9, A-1060 Wien, und

Institut für Mineralogie, Kristallographie und Strukturchemie

der Technischen Universität Wien ${ }^{\mathrm{b}}$,

Getreidemarkt 9, A-1060 Wien

Eingegangen am 1. Oktober 1984

Die Herstellung des enantiomerenreinen Thiolactols 1 wird beschrieben. Bei der Acetalisierung mit racemischem 1-Phenylethanol ( $r a c-2)$ reagiert je nach Wahl der Reaktionsbedingungen entweder $(R)-2$ oder $(S)-2$ bevorzugt.

Carbohydrate-Model Compounds, I

Kinetic and Thermodynamic Effects in Acetal Formation of Enantiomerically Pure Thiolactols

The synthesis of the enantiomerically pure thiolactol 1 is described. Depending on reaction conditions either $(R)-2$ or $(S)-2$ reacts with preference in acetal formation with racemic 1-phenylethanol (rac-2).

Im Laufe von Untersuchungen mit dem „terpenoiden Kohlenhydrat“-Modell I wurde bei Acetalisierungsreaktionen eine ausgeprägte Enantiomer-Selektivität beobachtet ${ }^{1}$, aus der nach weiteren Untersuchungen eine Regel zur Bestimmung der Absolutkonfiguration von Alkyl-aryl-carbinolen abgeleitet wurde ${ }^{2}$. Als Ursache für diese Enantiomer-Selektivität werden Unterschiede in der thermodynamischen Stabilität der gebildeten diastereomeren Acetale angenommen.

Die bei der Acetalisierung von 1-Phenylethanol (rac-2) beobachtete bevorzugte Reaktion des $R$-Enantiomeren im Ausmaß von 2:1 wurde, wie in Schema 1 skizziert, auf eine besonders stark destabilisierende nichtbindende Wechselwirkung ${ }^{3)}$ im mit (S)-2 gebildeten Acetal (B in Schema 1) zurückgeführt.

Ziel der vorliegenden Arbeit war es, durch sterische Veränderungen des Lactolmodells einerseits die getroffenen Annahmen zu bestätigen und andererseits - besonders in Hinblick auf eine präparative Anwendung - die EnantiomerSelektivität zu erhöhen. Da bei Strukturmodifikationen durch Einführung von Substituenten die Gefahr besteht, daß die für Lactolmodelle unbedingt wünschenswerte Anomer-Selektivität verloren geht ${ }^{1)}$, wählten wir ein Modell 1, in dem

(C) VCH Verlagsgesellschaft mbH, D-6940 Weinheim, 1985

$0009-2940 / 85 / 0808-3299 \$ 02.50 / 0$ 
lediglich der Ringsauerstoff gegen das deutlich größere Schwefelatom ausgetauscht ist ${ }^{4}$. In diesem Fall sollte durch die Wechselwirkung zwischen dem Schwefelatom und dem Phenylring das mit (S)-2 gebildete Acetal (D in Schema 1) gegenüber dem $(R)$-2-Acetal C noch stärker destabilisiert sein als B gegenüber A. Daher war zu erwarten, daß 1 eine höhere Enantiomer-Selektivität aufweisen würde als sie I besitzt.

Schema1
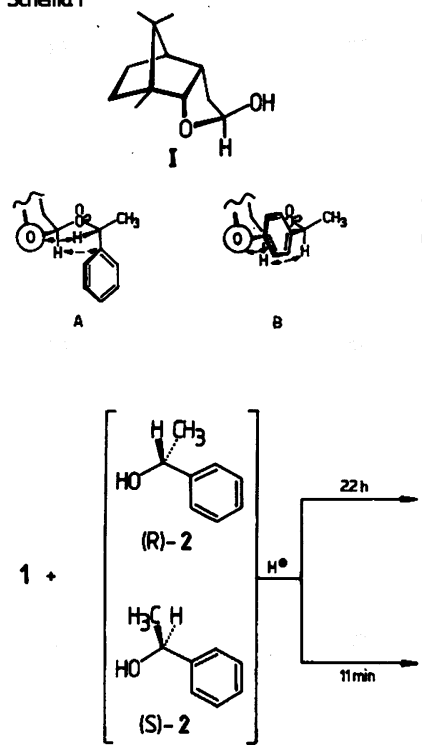

rac -2
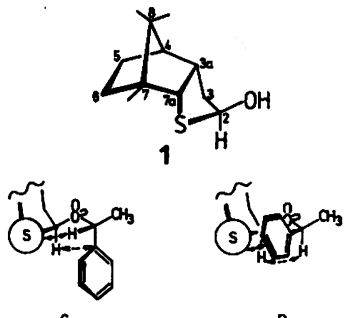

D

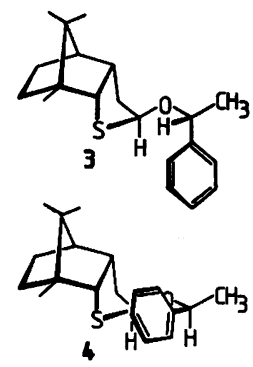

Untersuchungen mit der Verbindung 1 erschienen uns aber auch deshalb von Interesse, weil 5-Thio-D-glucose als Substrat für mechanistische Untersuchungen von Enzymreaktionen herangezogen wird ${ }^{5)}$, und 1 - in Analogie zum „terpenoiden Kohlenhydrat" I - als Modell eines "terpenoiden Thiozuckers" aufgefaßt werden kann.

\section{Herstellung des Thiolactols 1}

Ausgehend von Campheressigsäure 5 wurde durch Austausch der CampherCarbonylgruppe mit Lawesson-Reagens ${ }^{6}$ ) und anschließenden Ringschluß in einem Schritt das Thiolacton 6 in guter Ausbeute erhalten. Die Reduktion mit Natriumborhydrid führte zum Thiolacton 7, dessen Struktur aufgrund der chemischen Verschiebungen der Kohlenstoffe C-5 und C- ${ }^{7}$ als gewünschtes endocis-anelliertes Isomeres festgelegt werden konnte.

Bei geeigneter Reaktionsführung konnte die Natriumborhydrid-Reduktion gleich zum Lactol 1 und weiter zur vollständig reduzierten Verbindung 8, jeweils als Hauptprodukt, geführt werden. Für größere Ansätze erwies sich jedoch die 
Isolierung von 7 und eine anschließende Reduktion mit Diisobutylaluminiumhydrid zu 1 als überlegen.

Schema 2

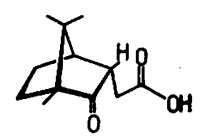

5
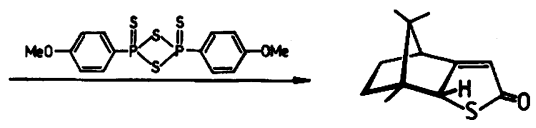

6

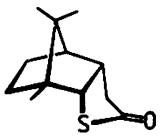

7
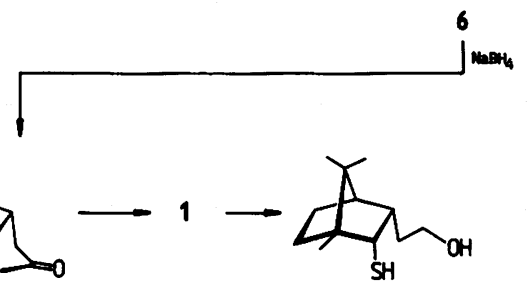

8

\section{Reaktives Verhalten von 1 in Gegenwart von Säure}

Während Lactole wie I bei Säurekatalyse unter Selbstacetalisierung ein symmetrisches Dimeres bilden, fand beim Thiolactol 1 unter gleichen Bedingungen eine Thioacetalbildung unter Ringöffnung zur dimeren Verbindung 9 statt. Bei Raumtemperatur war 9 nicht stabil und reagierte langsam in die Anhydroform $10 \mathrm{ab}$.

Schema 3
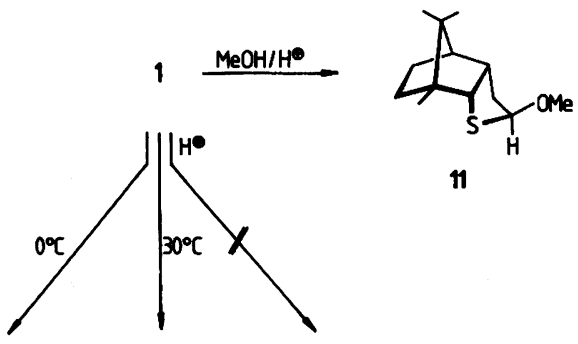

11

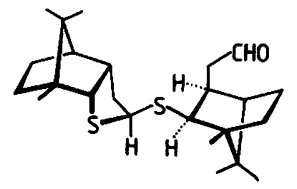

9<smiles>CC1(C)C2C=CCC1C1CCC2C1</smiles>

10

Die Acetalisierung von 1 mit überschüssigem Methanol bei Raumtemperatur führte direkt zum Methylacetal 11. Der relativ große Anteil an $\beta(e n d o)$-Anomerem $^{2,7}$, der bei dieser Verbindung beobachtet wurde $(\approx 15 \%)$, kam wegen des im Vergleich zum Sauerstoff schwächeren anomeren Effektes des Schwefels ${ }^{8)}$ keineswegs überraschend.

Chem. Ber. 118 (1985) 


\section{Kinetische und thermodynamische Steuerung bei der Enantiomer-selektiven Acetalisierung mit rac-2}

Zur Untersuchung in Hinblick auf die Enantiomer-Selektivität der Acetalisierung wurde 1 in einem NMR-Rohr-Versuch bei $0^{\circ} \mathrm{C}^{9)}$ mit überschüssigem rac-2 umgesetzt. Unmittelbar nach Zugabe der katalytischen Säuremengen wurden im Reaktionsgemisch, abgesehen von den Ausgangskomponenten, das Dimere 9 und zwei weitere Produkte beobachtet, welche aufgrund der Signallagen im Protonenkernresonanzspektrum als die gewünschten Thioacetale 3 und 4 angenommen wurden. Das aus den Integralen ermittelte Diastereomeren-Verhältnis war mit ungefähr 7:1 (nach 5 Minuten) tatsächlich wesentlich höher als jenes des Sauerstoffmodells $I(\approx 2: 1)$. Die spektroskopische Zuordnung der Absolutkonfiguration der Alkoxyreste (aufgrund von Abschirmungseffekten analog den entsprechenden Sauerstoff-Verbindungen ${ }^{1}$ ) ergab jedoch, da $B$ im Gegensatz zur ursprünglichen Erwartung das mit (S)-2 gebildete Diastereomere 4 bevorzugt gebildet worden war. Als Erklärung für diesen Befund bot sich eine kinetische Steuerung der Acetalisierung zum thermodynamisch instabileren Diastereomeren 4 an. Da die Ergebnisse der Untersuchungen über die Enantiomer-Selektivität der Sauerstoffmodelle unter Gleichgewichtsbedingungen erhalten worden waren, sollte sich bei längerer Reaktionszeit das Diastereomerenverhältnis in Richtung des thermodynamisch stabileren Diastereomeren 3 verlagern.
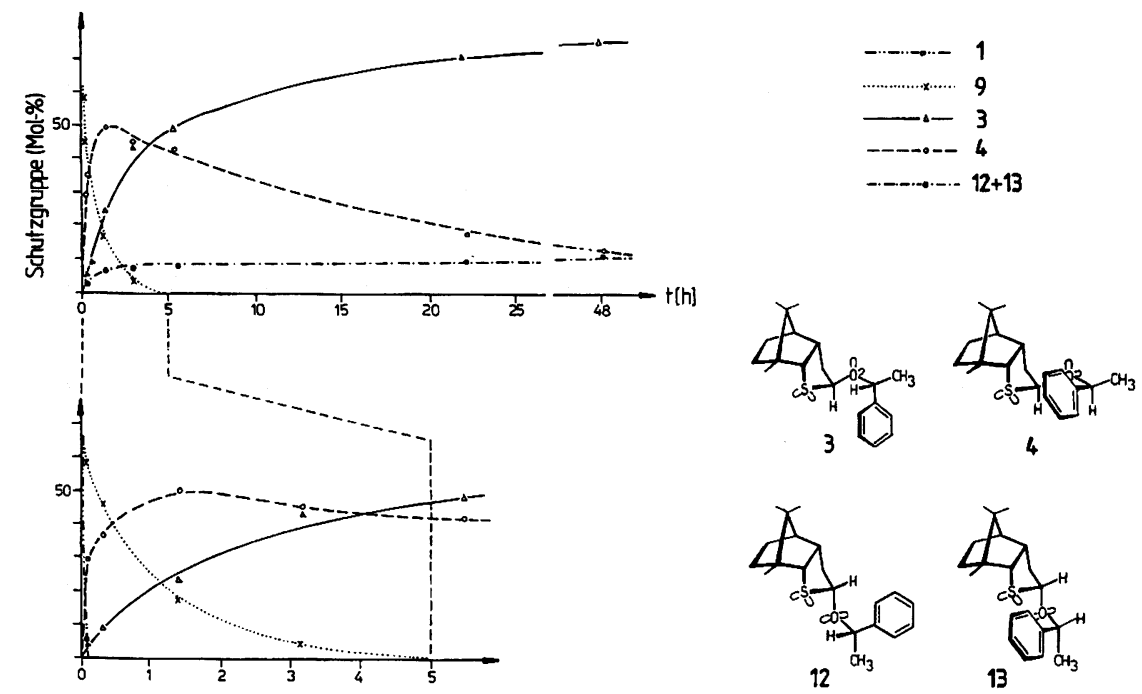

Abb. 1. Kinetische Untersuchung der Acetalisierung von 1 mit überschüssigem rac-2

Tatsächlich kam es im weiteren Verlauf des NMR-Versuchs relativ schnell zu einer Verschiebung des Diastereomeren-Verhältnisses 3:4, welches schließlich bei ungefähr 6:1 zugunsten von 3 konstant blieb. In einer eingehenden kinetischen Untersuchung wurden diese Befunde bestätigt (Abb. 1). 
Abgesehen von den geschilderten Ergebnissen kann aus der graphischen Darstellung die Entstehung und Abnahme des intermediär gebildeten 9, sowie der Anteil der gebildeten $\beta(e n d o)$-Anomeren 12 und 13 im Reaktionsgemisch abgelesen werden.

Da die bei der Reaktion entstehenden Produkte im Dünnschichtchromatogramm deutlich verschiedene $\boldsymbol{R}_{\mathrm{F}}$-Werte zeigten, wurde das gefundene Phänomen auch präparativ zur Enantiomer-selektiven Acetalisierung genutzt, wobei durch gezielten Abbruch der Reaktion entweder das kinetisch oder das thermodynamisch bevorzugte Produkt in überwiegender Menge säulenchromatographisch isoliert werden konnte.

So wurde die thermodynamisch gelenkte Acetalisierung nach 22 Stunden, die kinetisch gelenkte nach 11 Minuten gestoppt. Die Acetale 3, 4 und das $\beta(e n d o)$ Diastereomere 12 konnten rein isoliert und charakterisiert werden. Die quantitative säulenchromatographische Aufarbeitung der Reaktionsgemische bestätigte die Ergebnisse der NMR-Interpretation der kinetischen Untersuchung.

Zur weiteren Absicherung der Strukturen wurden aus 3 und 4 die enantiomerenreinen Alkohole $(R)-2$ und (S)-2 abgespalten. Da die aus 1 gebildeten Thioacetale in Gegenwart von Säure bei Raumtemperatur eine starke Neigung zur Bildung der Anhydroform gezeigt hatten, wurde die Abspaltung anstatt durch Methanolyse einfach durch Stehenlassen bei Raumtemperatur in Gegenwart von Toluolsulfonsäure bewirkt ${ }^{10)}$ und der bei der Lyse erhaltene Thioenolether 10 säulenchromatographisch abgetrennt.

\section{Konformationelle Betrachtungen anhand von Spektren und einer Röntgenstrukturanalyse}

Als Basis für die Betrachtungen wurden - wie oben erwähnt - die Abschirmungseffekte in den Protonenresonanzspektren der diastereomeren Acetale herangezogen ${ }^{1}$. Die bei 3 und 4 beobachteten Abschirmungseffekte entsprachen weitgehend den bei den analogen Verbindungen des Sauerstoffmodells I gefundenen Ergebnissen: so liegen bei 3 die Signale der Protonen an C-2 um $\Delta \delta=$ $0.4 \mathrm{ppm}$ bei höherem Feld, die der Protonen an C-7a um $\Delta \delta=0.12 \mathrm{ppm}$ bei tieferem Feld als bei $4^{11}$.

Um einen noch besseren Vergleich zwischen Sauerstoffmodell I und Schwefelmodell 1 zu haben, wurde, analog zu Modell I, das (R)-1-Phenylethylacetal 3 einer Röntgenstrukturanalyse unterzogen.

\section{Röntgenstrukturanalyse von 3}

Ein prismatischer Einkristall mit den Abmessungen $0.014 \times 0.024 \times 0.039 \mathrm{~cm}$ wurde zur Strukturermittlung auf einem Vierkreisdiffraktometer mit Mo- $K_{\alpha}$ Strahlung vermessen (PW 1100 mit Graphitmonochromator). Für die orthorhombische Elementarzelle wurden folgende kristallographische Daten erhalten: $a=$ 1946.1(2), $b=1274.6(1)$ und $c=734.76(5) \mathrm{pm}$; Raumgruppe $P 22_{1} 2_{1} 2_{1}$ (Nr. 19); $Z=4$ und $D_{\mathrm{x}}=1.153 \mathrm{gcm}^{-3}$. Die Reflexintensitäten wurden mit $\omega / 2 \Theta$-Scans im Bereich bis $\omega=26^{\circ}$ gesammelt. Von den 2056 unabhängigen, nicht ausge- 
Tab. 1. Atomkoordinaten und Temperaturparameter (in $\mathrm{pm} \times 10^{-4}$ ) von 3 mit Standardabweichungen der letzten Stelle in Klammern; $U_{\text {iso }}$ für die Wasserstoffatome betragen 0.073(5) $\left(\mathrm{CH}\right.$ und $\left.\mathrm{CH}_{2}\right)$ und $0.076(5)\left(\mathrm{CH}_{3}\right)$

\begin{tabular}{|c|c|c|c|c|c|c|c|c|}
\hline atom & $\times$ & $y$ & $=$ & $v_{100}$ & Atom & $\mathbf{x}$ & $y$ & $z$ \\
\hline$c 2$ & $0.6304(2)$ & $0.4351(4)$ & $0.616(1)$ & $0.046(1)$ & B21 & 0.627 & 0.514 & 0.564 \\
\hline c3 & $0.6769(3)$ & $0.3636(4)$ & $0.507(1)$ & $0.047(1)$ & 831 & 0.652 & 0.341 & 0.382 \\
\hline c3a & $0.6918(2)$ & $0.2665(4)$ & $0.623(1)$ & $0.040(1)$ & H32 & 0.724 & 0.404 & 0.476 \\
\hline C4 & $0.7627(2)$ & $0.2113(4)$ & $0.601(1)$ & $0.039(1)$ & H. 3 I & 0.652 & 0.213 & 0.576 \\
\hline cs & $0.8200(3)$ & $0.2928(4)$ & $0.598(1)$ & $0.049(1)$ & H41 & 0.764 & 0.161 & 0.482 \\
\hline c6 & $0.8180(3)$ & $0.3356(4)$ & $0.793(1)$ & $0.051(2)$ & H51 & 0.869 & 0.257 & 0.568 \\
\hline c7 & $0.7649(3)$ & $0.2671(4)$ & $0.891(1)$ & $0.047(1)$ & В52 & 0.809 & 0.354 & 0.500 \\
\hline c7a & $0.6919(2)$ & $0.2973(4)$ & $0.827(1)$ & $0.038(1)$ & 861 & 0.802 & 0.417 & 0.793 \\
\hline co & $0.7722(3)$ & $0.1606(4)$ & $0.792(1)$ & $0.046(1)$ & H62 & 0.868 & 0.328 & 0.857 \\
\hline c9 & $0.7734(4)$ & $0.2677(6)$ & $1.098(1)$ & $0.073(2)$ & B7al & 0.656 & 0.258 & 0.915 \\
\hline c10 & $0.7188(3)$ & $0.0771(5)$ & $0.837(1)$ & $0.066(2)$ & 891 & 0.824 & 0.242 & 1.142 \\
\hline c1.1 & $0.8436(3)$ & $0.1074(5)$ & $0.820(1)$ & $0.063(2)$ & н92 & 0.736 & 0.213 & 1.146 \\
\hline $\mathrm{C} 12$ & $0.5114(3)$ & $0.4689(4)$ & $0.659(1)$ & $0.050(1)$ & н93 & 0.763 & $0.34 \mathrm{~s}$ & 1.152 \\
\hline$c 13$ & $0.4459(3)$ & $0.4067(5)$ & $0.698(1)$ & $0.068(2)$ & H101 & 0.668 & 0.099 & 0.789 \\
\hline c14 & $0.5003(3)$ & $0.5495(4)$ & $0.513(1)$ & $0.043(1)$ & H102 & 0.718 & 0.072 & 0.984 \\
\hline c1s & $0.5059(3)$ & $0.5241(5)$ & $0.330(1)$ & $0.064(2)$ & H103 & 0.732 & 0.002 & 0.781 \\
\hline$c 16$ & $0.4937(3)$ & $0.5978(6)$ & $0.194(1)$ & $0.078(2)$ & H111 & 0.887 & 0.159 & 0.811 \\
\hline $\mathrm{C17}$ & $0.4745(3)$ & $0.6981(5)$ & $0.239(1)$ & $0.072(2)$ & H112 & 0.848 & 0.046 & 0.719 \\
\hline $\operatorname{c1s}$ & $0.4692(3)$ & $0.7257(5)$ & $0.416(1)$ & $0.062(2)$ & H113 & 0.840 & 0.074 & 0.954 \\
\hline c19 & $0.4825(3)$ & $0.6517(5)$ & $0.554(1)$ & $0.057(2)$ & 8121 & 0.527 & 0.513 & 0.778 \\
\hline$\circ$ & $0.5628(2)$ & $0.3940(3)$ & $0.6092(5)$ & $0.047=$ & H131 & 0.408 & 0.465 & 0.732 \\
\hline \multirow[t]{6}{*}{$s$} & $0.6663(1)$ & $0.4349(1)$ & $0.8453(2)$ & $0.054^{\circ}$ & 8132 & 0.448 & 0.349 & 0.806 \\
\hline & & & & & 8133 & 0.432 & 0.368 & 0.573 \\
\hline & & & & & H151 & 0.520 & 0.445 & 0.292 \\
\hline & & & & & 8161 & 0.499 & 0.576 & 0.053 \\
\hline & & & & & B171 & 0.464 & 0.755 & 0.133 \\
\hline & & & & & H1B1 & 0.455 & 0.805 & 0.452 \\
\hline - aus & anisotropen & Parametern ber & net & & H191 & 0.479 & 0.675 & 0.695 \\
\hline
\end{tabular}

löschten Reflexen wiesen 1466 Intensitäten $\geqslant 2 \sigma(I)$ auf. Ein Lösungsversuch mit Direkten Methoden (MULTAN 78 ${ }^{12}$ ) lieferte 18 der 22 schweren Atome, die restlichen 4 konnten mit Hilfe einer Differenz-Fourier-Synthese lokalisiert werden. Die Verfeinerung der Struktur erfolgte nach der Methode der kleinsten Quadrate (SHELX $76^{13}$ ), wobei die Wasserstoffatome auf geometrisch ermittelten Lagen eingesetzt wurden (C-H-Abstand $108 \mathrm{pm}$ ). Die Lageparameter der Wasserstoffatome wurden stets zusammen mit den bindenden Kohlenstoffatomen variiert, lediglich die vier Methylgruppen besaßen als zusätzliche Freiheitsgrade die Möglichkeit der freien Rotation als starre Gruppen. Für das Sauerstoff- und Schwefelatom wurden anisotrope, für die Kohlenstoff- und Wasserstoffatome isotrope Temperaturfaktoren eingesetzt. Die Verfeinerung mit allen 2056 unabhängigen Reflexen führte auf die in Tab. 1 angeführten Parameter und die $R$-Werte: $R=$ 0.097 und $R_{w}=0.081$ mit $w=1 /\left(\sigma^{2}(F)+0.0005 F^{2}\right)^{*)}$.

*) Weitere Einzelheiten zur Kristallstrukturuntersuchung können beim Fachinformationszentrum Energie Physik Mathematik, D-7514 Eggenstein-Leopoldshafen, unter Angabe der Hinterlegungsnummer CSD 511 54, des Autors und des Zeitschriftenzitats angefordert werden. 
Die Konformation des untersuchten Thioacetals 3 stimmt im wesentlichen mit jener der analogen Sauerstoffverbindung überein. Als markantester Unterschied fält zunächst die stärkere Knickung des fünfgliedrigen Thiolactolringes auf $\left(41^{\circ}\right.$ gegenüber $31^{\circ}$ bei der Sauerstoffverbindung). Der chirale Alkoxyrest weist die erwartete, durch den exo-anomeren Effekt stabilisierte Konformation auf, weicht jedoch mit einem Torsionswinkel von $81.8^{\circ}$ (Tab. 2) deutlich stärker von der idealen Anordnung mit $60^{\circ}$ ab als die Sauerstoffverbindung mit $74.3^{\circ 14}$. Auch die Phenylgruppe ist mit $73.5^{\circ}$ stärker im Gegenuhrzeigersinn aus der gestaffelten Anordnung herausgedreht als bei der Sauerstoffverbindung mit $68.7^{\circ}$. Beide Drehungen führen zu einer Vergrößerung des Abstandes der Phenylgruppe vom Schwefelatom.

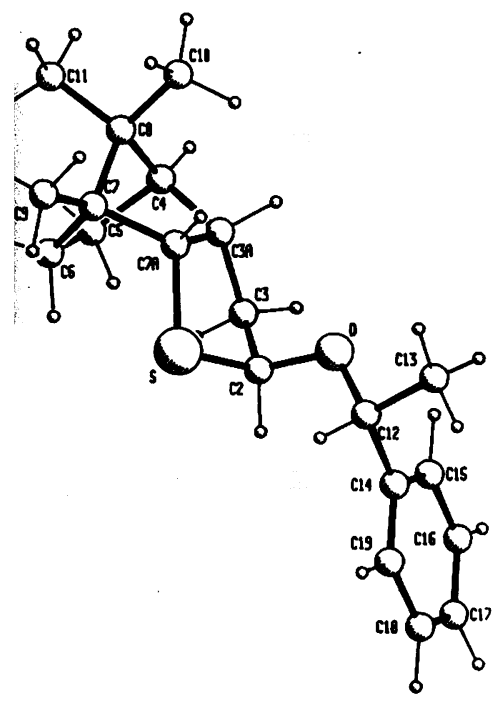

Abb. 2. Molekülstruktur von 3
Tab. 2. Ausgewählte interatomare Abstände, Winkel und Torsionswinkel der Verbindung 3

\begin{tabular}{|c|c|c|c|c|c|}
\hline Abstánde & {$[\mathrm{pm}]$} & winkel $[\cdot]$ & & Torsionswinkel & {$[\cdot]$} \\
\hline$s-c_{2}$ & $182.5(5)$ & $c 7 a-s-c 2$ & $92.2(2)$ & $c 7 a-s-c 2-c 3$ & -35.1 \\
\hline$s-c 7 a$ & $182.8(5)$ & $s-c 2-c 3$ & $104.8(3)$ & $s-c 2-c 3-c 3 a$ & 43.5 \\
\hline$c 2-c 3$ & $151.2(7)$ & $0-c 2-s$ & $112.8(4)$ & $c 2-c 3-c 3 a-c 7 a$ & -31.4 \\
\hline$c 3-c 3 a$ & $152.7(7)$ & $0-c 2-c 3$ & $108.4(4)$ & $c 3-c 3 a-c 7 a-s$ & 5.1 \\
\hline$c 3 a-c 7 a$ & $155.5(7)$ & $c 2-c 3-c 3 a$ & $108.1(4)$ & $c 3 a-c 7 a-s-c 2$ & 17.4 \\
\hline$c 3 a-c 4$ & $155.7(6)$ & $c 3-c 3 a-c 4$ & $118.5(4)$ & $c 7 a-s-c 2-0$ & 82.6 \\
\hline C4-c8 & $155.2(7)$ & $c 3-c 3 a-c 7 a$ & $109.4(4)$ & c12-0-c2-s & 81.8 \\
\hline c4-cs & $152.4(7)$ & $c 4-c 3 a-c 7 a$ & $102.2(4)$ & $c 12-0-c 2-c 3$ & -162.4 \\
\hline cs-c6 & $153.3(7)$ & $c 3 a-c 4-c 5$ & $130.0(4)$ & $C 13-c 12-0-C_{2}$ & -164.1 \\
\hline$c 6-c 7$ & $153.2(7)$ & $c 4-c 5-c 6$ & $102.0(4)$ & $c 14-c 12-0-c 2$ & 73.5 \\
\hline$c 7-c 7 a$ & $154.4(7)$ & $\mathrm{cs}-\mathrm{Cb}-\mathrm{Cl}$ & $104.7(4)$ & C15-C14-C12-o & 34.2 \\
\hline$c 7-c 8$ & $154.8(7)$ & $\mathrm{c} 6-\mathrm{c} 7-\mathrm{C7a}$ & $109.7(4)$ & c19-c14-c12-o & -147.3 \\
\hline$c 7-c 9$ & $152.9(8)$ & $C 4-C 8-c 7$ & $92.8(4)$ & & \\
\hline$c 8-c 10$ & $152.4(7)$ & $c 7-c 7 a-s$ & $117.9(3)$ & & \\
\hline$c 8-c 11$ & $156.0(7)$ & $c 7-c 7 a-c 3 a$ & $103.3(4)$ & & \\
\hline c2-o & $141.7(6)$ & $c 3 a-c 7 a-s$ & $108.2(3)$ & & \\
\hline $0-\operatorname{cis}$ & $143.2(6)$ & $c 2-0-c 12$ & $113.1(4)$ & & \\
\hline$c 12-c 13$ & $152.8(8)$ & $0-c 12-c_{13}$ & $106.5(4)$ & & \\
\hline \multirow[t]{2}{*}{ C12-C14 } & $150.0(7)$ & $0-c 12-c 14$ & $111.9(4)$ & & \\
\hline & & $C 13-c 12-c 14$ & $111.7(5)$ & & \\
\hline
\end{tabular}

Der im Vergleich zum Sauerstoffanalogon erhöhte Anteil an $\beta(e n d o)$-Anomerem ermöglichte eine Untersuchung der beiden $\beta(e n d o)$-Acetale 12 und 13. Die Zuordnung der $\beta(e n d o)$-Anomeren erfolgte aufgrund des $\gamma$-Effektes in den ${ }^{13} \mathrm{C}$-Kernresonanzspektren, in welchen die Lagen von C-3a und C-7a in den $\alpha(e x o)$-Anomeren bei höherem Feld auftreten'). Wie erwartet, bewirkten die chiralen Alkoxyreste von 12 und 13 eine im Vergleich zu den $\alpha(e x o)$-Anomeren umgekehrte Richtung der Abschirmungseffekte: Die Signallagen der Protonen an C-2 des (S)-Diastereomeren 12 liegen um $\Delta \delta=0.4 \mathrm{ppm}$ bei höherem Feld als jene des $(R)$-Diastereomeren 13. Auch das beim Langzeitversuch erhaltene Produktverhältnis 12:13 war mit 3:1 im Vergleich zu den (exo)-Anomeren entgegengesetzt gerichtet.

Aus den bisherigen Ergebnissen lassen sich die unter Gleichgewichtsbedingungen gefundenen Enantiomer-Selektivitäten erklären ${ }^{15)}$. Die Tatsache, daß es unter kinetischen Bedingungen zu einer Umkehrung der Enantiomer-Selektivität 
kommt, wird Gegenstand weiterer Untersuchungen sein. Die Ergebnisse dieser Arbeit zeigen, daß das „terpenoide Kohlenhydrat" I und der „terpenoide. Thiozucker" 1 - und somit mit großer Wahrscheinlichkeit auch Zucker und Thiozucker - nicht nur im Hinblick auf die Reaktionsgeschwindigkeit, sondern auch im Hinblick auf die Reaktionsprodukte Unterschiede aufweisen.

Diese Arbeit wurde durch Mittel aus der Hochschuljubiläumsstiftung der Stadt Wien gefördert.

\section{Experimenteller Teil}

Allgemeine Angaben: vgl. Lit. ${ }^{1,7)}$.

Abkürzungen: PE $=$ Petrolether, $\mathrm{E}=$ Ether, $\mathrm{MBT}=[2 S-(2 \alpha, 3 \mathrm{a} \alpha, 4 \alpha, 7 \alpha, 7 \mathrm{a} \alpha)]-$ Octahydro-7,8,8-trimethyl-4,7-methanobenzo[b]thien-2-yl.

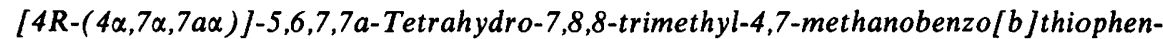
2(4H)-on (6): $1.00 \mathrm{~g}$ (4.76 mmol) Campheressigsäure $5^{7}$ und $1.15 \mathrm{~g}$ (2.85 mmol) 2,4-Bis(4methoxyphenyl)-1,3,2,4-dithiadiphosphetan-2,4-disulfid ${ }^{6}$ wurden in $15 \mathrm{ml}$ wasserfreiem Toluol $2 \mathrm{~h}$ unter Stickstoff bei $105^{\circ} \mathrm{C}$ gerührt. Es wurde mit E/PE (1:1) verdünnt. Nach Abkühlen wurde die Lösung filtriert und zwischen $\mathrm{E}$ und Natriumhydrogencarbonatlösung verteilt; die Extrakte wurden gewaschen und i. Vak. eingedampft. $0.86 \mathrm{~g}$ Rohprodukt wurden durch SC ( $25 \mathrm{~g}$ Kieselgel feinst, Eluens: $\mathrm{PE} / \mathrm{E}=15: 1)$ gereinigt und ergaben $0.65 \mathrm{~g}(66 \%)$ 6. Farblose Kristalle, Schmp. $45-46^{\circ} \mathrm{C}$ (aus PE), $[\alpha]_{\mathrm{D}}^{22}=-158^{\circ}(c=0.98$ in $n$-Hexan). IR (KBr): $2950(\mathrm{CH}), 1685(\mathrm{C}=\mathrm{O}), 1630 \mathrm{~cm}^{-1}(\mathrm{C}=\mathrm{C}) .-{ }^{1} \mathrm{H}-\mathrm{NMR}\left(\mathrm{CDCl}_{3}\right): \delta=5.95(\mathrm{~d}$, $J=2.5 \mathrm{~Hz} ; 1 \mathrm{H}, 3-\mathrm{H}), 4.68(\mathrm{~s}, \mathrm{br} ; 1 \mathrm{H}, 7 \mathrm{a}-\mathrm{H}), 3.01$ (d, $J=3.9 \mathrm{~Hz} ; 1 \mathrm{H}, 4-\mathrm{H}), 2.26-1.10(\mathrm{~m}$; 4H, 5-H, 6-H), 1.01 (s; 6H, 2 $\left.\mathrm{CH}_{3}\right), 0.99\left(\mathrm{~s} ; 3 \mathrm{H}, \mathrm{CH}_{3}\right) .-{ }^{13} \mathrm{C}-\mathrm{NMR}\left(\mathrm{CDCl}_{3}\right): \delta=200.6(\mathrm{~s} ;$ C-2), 190.7 (s; C-3a), 123.4 (d; C-3), 65.2 (d; C-7a), 53.9 (d; C-4), 52.1 (s; C-8), 49.6 (s; C-7), 35.4 (t; C-6), 27.4 (t; C-5), 19.6 (q; $\mathrm{CH}_{3}$ ), 19.5 (q; $\mathrm{CH}_{3}$ ), 14.7 (q; $\mathrm{CH}_{3}$ ).

$$
\mathrm{C}_{12} \mathrm{H}_{16} \mathrm{OS}(208.3) \quad \text { Ber. C } 69.19 \text { H } 7.74 \text { Gef. C } 69.23 \text { H } 7.72
$$

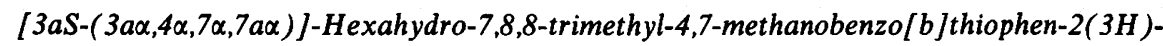
on (7): $5.80 \mathrm{~g}$ (27.8 mmol) 6 in $250 \mathrm{ml}$ Ethanol wurden mit $1.06 \mathrm{~g}(27.8 \mathrm{mmol})$ Natriumborhydrid $30 \mathrm{~min}$ bei Raumtemp. gerührt. Es wurde Natriumhydrogencarbonatlösung zugegeben, mit PE/E extrahiert, mit Natriumsulfat getrocknet und i. Vak. eingedampft. Das Rohprodukt wurde durch SC aufgetrennt: $3.99 \mathrm{~g}(68 \%) 7,1.35 \mathrm{~g} \mathrm{(23 \% )} 1$ und $0.24 \mathrm{~g} \mathrm{(4 \% )} 8$.

7: Farblose Nadeln, Schmp. $45^{\circ} \mathrm{C}$ (aus PE/E). $-[\alpha]_{\mathrm{D}}^{20}=+93^{\circ}(c=1.06$ in Chloroform). - IR (KBr): $2950(\mathrm{CH}), 1700(\mathrm{C}=\mathrm{O}), 1050,1025 \mathrm{~cm}^{-1} .-{ }^{1} \mathrm{H}-\mathrm{NMR}\left(\mathrm{CDCl}_{3}\right): \delta=$ $4.08(\mathrm{~d}, J=10.7 \mathrm{~Hz} ; 1 \mathrm{H}, 7 \mathrm{a}-\mathrm{H}), 3.26-2.87(\mathrm{~m} ; 1 \mathrm{H}, 3 \mathrm{a}-\mathrm{H}), 2.87-2.30(\mathrm{~m} ; 2 \mathrm{H}, 3-\mathrm{H})$, 2.19-1.10 (m; 5H, Aliphaten-H), $1.02\left(\mathrm{~s} ; 6 \mathrm{H}, 2 \mathrm{CH}_{3}\right), 0.85\left(\mathrm{~s} ; 3 \mathrm{H}, \mathrm{CH}_{3}\right) .-{ }^{13} \mathrm{C}-\mathrm{NMR}$ $\left(\mathrm{CDCl}_{3}\right): \delta=210.3$ (s; C-2), 59.9 (d; C-7a), 50.2 (s; C-8), 49.5 (d; C-4), 49.1 (s; C-7), 43.2 (t; C-3), 37.5 (d; C-3a), 29.4 (t; C-6), 20.6 (q; $\mathrm{CH}_{3}$ ), 20.2 (t; C-5), 18.2 (q; $\mathrm{CH}_{3}$ ), 16.0 (q; $\mathrm{CH}_{3}$ ).

$$
\mathrm{C}_{12} \mathrm{H}_{18} \mathrm{OS}(210.3) \text { Ber. C } 68.52 \text { H } 8.63 \text { Gef. C } 68.66 \text { H } 8.57
$$

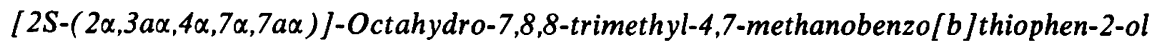
$(\mathbf{1}=\mathrm{MBT}-\mathrm{OH})$

a) Durch $\mathrm{NaBH}_{4}$-Reduktion von 6: $2.00 \mathrm{~g}(9.62 \mathrm{mmol}) 6$ in $30 \mathrm{ml}$ Ethanol wurden mit $0.73 \mathrm{~g}(19.24 \mathrm{mmol})$ Natriumborhydrid $3.5 \mathrm{~h}$ bei Raumtemp. gerührt. Das nach Aufarbeitung wie bei 7 erhaltene Produktgemisch wurde durch SC aufgetrennt in $0.59 \mathrm{~g} \mathrm{(29 \% )} \mathrm{7,} 0.88 \mathrm{~g}$ (43\%) 1 und $0.32 \mathrm{~g}(16 \%) 8$ (7:1:8 = 33:49:18). $M B T-O H$ (1): Farblose Kristalle, Schmp. $43-45^{\circ} \mathrm{C}$ (aus PE): Sdp. $100^{\circ} \mathrm{C} / 0.02$ Torr (Luftbad). $-[\alpha]_{\mathrm{D}}^{20}=+156^{\circ}$ (c $=0.48$ in 
Chlorroform). - IR (KBr): 3350 (breite Bande, OH), 2940 (CH), 1035, $1005 \mathrm{~cm}^{-1}$. - ${ }^{1} \mathrm{H}$ NMR $\left(\mathrm{CDCl}_{3}\right): \delta=5.71(\mathrm{t}, J=8.6 \mathrm{~Hz} ; 1 \mathrm{H}, 2-\mathrm{H}), 4.04 / 4.01 / 3.92 / 3.89$ (dd; $1 \mathrm{H}, 7 \mathrm{a}-\mathrm{H}$ ), $3.32-2.65$ (m; 2H, 3a-H, OH), 2.16-1.06 (m; 7H, Aliphaten-H), 1.04/0.96/0.76 (3 s; $9 \mathrm{H}$, $\left.3 \mathrm{CH}_{3}\right) .-{ }^{13} \mathrm{C}-\mathrm{NMR}\left(\mathrm{CDCl}_{3}\right): \delta=87.2(\mathrm{~d} ; \mathrm{C}-2), 61.4$ (d; C-7a), 52.3 (s; C-7), 49.4 (s; C-8), 48.2 ((d; C-4), 45.0 (d; C-3a), 40.6 (t; C-3), 28.8 (t; C-6), 21.2 (t, q; C-5, $\mathrm{CH}_{3}$ ), 18.6 (q; $\mathrm{CH}_{3}$ ), $15.8\left(\mathrm{q} ; \mathrm{CH}_{3}\right)$.

\section{$\mathrm{C}_{12} \mathrm{H}_{20} \mathrm{OS}$ (212.3) Ber. C 67.87 H 9.49 Gef. C 67.76 H 9.34}

b) Aus 7 durch Reduktion mit ( $\mathrm{BBu})_{2} \mathrm{AlH}: \mathrm{Zu} 2.5 \mathrm{~g}(11.9 \mathrm{mmol}) 7 \mathrm{in} 25 \mathrm{ml}$ wasserfreiem Toluol wurden bei $-40^{\circ} \mathrm{C} 10.9 \mathrm{ml}(13.1 \mathrm{mmol})$ einer 20proz. Lösung von Diisobutylaluminiumhydrid in $n$-Hexan getropft und $1 \mathrm{~h}$ bei $-40^{\circ} \mathrm{C}$ gerührt. Nach Zugabe von $20 \mathrm{ml} \mathrm{E}$ und $10 \mathrm{ml}$ Wasser wurde bei Raumtemp. $2 \mathrm{~N} \mathrm{NaOH}$ bis zur Auflösung des Aluminiumhydroxiid-Niederschlags zugesetzt, die organische Phase abgetrennt und mit $\mathrm{E}$ extrahiert; die vereimigten organischen Phasen wurden gewaschen. Trocknen und Eindampfen i. Vak. ergab $2.5 \mathrm{~g}(99 \%) 1$.

[1R-(endo,endo)]-3-Mercapto-4,7,7-trimethylbicyclo[2.2.1] heptan-2-ethanol (8): Als Nebenprodukt bei der $\mathrm{NaBH}_{4}$-Reduktion von 6 (siehe Herstellung von 7 bzw. 1). Farbloses Öl, Sidp. $80^{\circ} \mathrm{C} / 0.02$ Torr (Luftbad); $[\alpha]_{\mathrm{D}}^{20}=+29^{\circ}(c=0.80$ in Chloroform). - IR (KBr): 3340 (breite Bande, $\mathrm{OH}), 2550(\mathrm{SH}), 1050 \mathrm{~cm}^{-1}(\mathrm{C}-\mathrm{O}) .-{ }^{1} \mathrm{H}-\mathrm{NMR}\left(\mathrm{CDCl}_{3}\right): \delta=3.58(\mathrm{t}$; $\left.J=7 \mathrm{~Hz} ; 2 \mathrm{H}, \mathrm{CH}_{2}-\mathrm{O}\right), 3.45-3.13(\mathrm{~m} ; 1 \mathrm{H}, \mathrm{CH}-\mathrm{S}), 2.52-2.1$ (br. s; $\left.2 \mathrm{H}, \mathrm{OH}, \mathrm{SH}\right)$, 2.0-0.98 (m; $8 \mathrm{H}$, Aliphaten-H), $0.94\left(\mathrm{~s} ; 6 \mathrm{H}, 2 \mathrm{CH}_{3}\right), 0.82\left(\mathrm{~s} ; 3 \mathrm{H}, \mathrm{CH}_{3}\right) .-{ }^{13} \mathrm{C}-\mathrm{NMR}\left(\mathrm{CDCl}_{3}\right)$ : $\delta=62.0(\mathrm{t} ; \mathrm{C}-\alpha), 50.6$ (s; C-4), 48.9 (d; C-1), 48.6 (d; C-3), 47.2 (s; C-7), 35.4 (d; C-2), 32.1 (t; C- $\beta$ ), 27.9 (t; C-5), 20.4 (q; $\mathrm{CH}_{3}$ ), 19.9 (t; C-6), 18.0 (q, $\left.\mathrm{CH}_{3}\right), 14.4$ (q; $\mathrm{CH}_{3}$ ).

\section{$\mathrm{C}_{12} \mathrm{H}_{22} \mathrm{OS}$ (214.4) Ber. C 67.23 H 10.34 Gef. C 67.48 H 10.29}

[1R-[endo,endo $\left.\left.\left(2^{\prime} R^{*}, 3^{\prime} a R^{*}, 4^{\prime} R, 7^{\prime} S^{*}, 7^{\prime} a R^{*}\right)\right]\right]-3-[($ Octahydro-7,8,8-trimethyl-4,7-methanobenzo[ $b$ ] thien-2-yl)thio]-4,7,7-trimethylbicyclo[2.2.1] heptan-2-ethanal (9): $83 \mathrm{mg}(0.39$ mmol) 1 wurden in $0.5 \mathrm{ml} \mathrm{CDCl}$ bei $0^{\circ} \mathrm{C}$ mit $1 \mathrm{mg} p$-Toluolsulfonsäure 15 min stehengelassen. Durch Zugabe von $3 \mu$ T Triethylamin wurde die Reaktion gestoppt, festes Natriumhydrogencarbonat und Natriumsulfat zum Trocknen zugegeben, filtriert und i. Vak. eingedampft; $71 \mathrm{mg}(90 \%)$ 9; farblose Kristalle, Schmp. $73-75^{\circ} \mathrm{C}(\mathrm{PE}) ;[\alpha]_{\mathrm{D}}^{20}=+173^{\circ}(\mathrm{c}=$ 1.30 in Tetrachlormethan). - IR (KBr): 2945, 2925 (CH), $1720 \mathrm{~cm}^{-1}$ (C=O). - ${ }^{1} \mathrm{H}-\mathrm{NMR}$ $\left(\mathrm{CDCl}_{3}\right): \delta=9.90$ (s; $1 \mathrm{H}, \mathrm{CHO}$ ), 4.41/4.34/4.31/4.24 (dd; $\left.1 \mathrm{H}, 2-\mathrm{H}\right), 3.78$ (d, $J=10.4 \mathrm{~Hz}$ $1 \mathrm{H}, 7 \mathrm{a}-\mathrm{H}), 3.27-1.0(\mathrm{~m} ; 17 \mathrm{H}$, Aliphaten-H), 1.02/0.98/0.95/0.92/0.92/0.74 $(6 \mathrm{~s} ; 18 \mathrm{H}$, 6. $\left.\mathrm{CH}_{3}\right) .-{ }^{13} \mathrm{C}-\mathrm{NMR}\left(\mathrm{CDCl}_{3}\right): \delta=202.1$ (d; CHO), 62.8 (d; C-2), 59.1 (d; C-7a), 57.4 (d; C-3'), 51.4 (s; C-7), 50.7 (s; C-4'), 50.1 (s; C-8), 49.3 (d; C-4), 49.2 (d; C-1'), 47.7 (s; C-7'), 46.6 (d; C-3a), 45.2 (t; $\mathrm{CH}_{2} \mathrm{CHO}$ ), 39.9 (t; C-3), 35.3 (d; C-2'), 29.6 (t; C-6), 29.2 (t; C-5'), 21.5 (t; C-5), 20.6 (q; $\mathrm{CH}_{3}$ ), 20.4 (t; C-6'), 20.0 (q; $\left.\mathrm{CH}_{3}\right), 18.6$ (q; $\left.\mathrm{CH}_{3}\right), 18.4$ (q; $\mathrm{CH}_{3}$ ), 15.7 (q; $\mathrm{CH}_{3}$ ), $14.8\left(\mathrm{q} ; \mathrm{CH}_{3}\right)$.

$$
\mathrm{C}_{24} \mathrm{H}_{38} \mathrm{OS}_{2} \text { (406.7) Ber. C } 70.88 \text { H } 9.42 \text { Gef. C } 70.74 \text { H } 9.36
$$

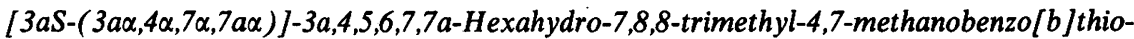
phen (10): $129 \mathrm{mg}(0.61 \mathrm{mmol}) 1$ wurden in einem NMR-Versuch in $\mathrm{CDCl}_{3}$ bei $30^{\circ} \mathrm{C}$ mit $p$ Toluolsulfonsäure zur Katalyse versetzt; nach 10 min bestand das Reaktionsgemisch zu mehr als $80 \%$ aus 9, ein kleiner Anteil an 10 war vorhanden. Nach $24 \mathrm{~h}$ war die Umsetzung zu 10 vollständig. Nach Zugabe von festem Natriumhydrogencarbonat und Natriumsulfat wurde filtriert, vorsichtig eingedampft (10 ist relativ flüchtig!) und $77 \mathrm{mg}(65 \%) 10$ isoliert. Farbloses Öl, Sdp. $55^{\circ} \mathrm{C} / 0.5$ Torr (Luftbad); $[\alpha]_{\mathrm{D}}^{22}=+75^{\circ}(c=0.66$ in Dichlormethan). IR (KBr): $2940 \mathrm{~cm}^{-1}$ (CH). - ${ }^{1} \mathrm{H}-\mathrm{NMR}\left(\mathrm{CDCl}_{3}\right): \delta=6.07 / 6.05 / 6.00 / 5.98$ (dd; $\left.1 \mathrm{H}, 2-\mathrm{H}\right)$, $5.30 / 5.27 / 5.23 / 5.20(\mathrm{dd} ; 1 \mathrm{H}, 3-\mathrm{H}), 4.1\left(\mathrm{~d}_{\mathrm{AB}}, J_{\mathrm{AB}}=12 \mathrm{~Hz} ; 1 \mathrm{H}, 7 \mathrm{a}-\mathrm{H}\right), 3.7\left(\mathrm{~d}_{\mathrm{AB}}, J_{\mathrm{AB}}=12 \mathrm{~Hz}\right.$; 
$1 \mathrm{H}, 3 \mathrm{a}-\mathrm{H}), 2.19-1.04\left(\mathrm{~m} ; 5 \mathrm{H}\right.$, Aliphaten-H), 1.01/0.96/0.78 (3 s; $\left.9 \mathrm{H}, 3 \mathrm{CH}_{3}\right) .-{ }^{13} \mathrm{C}-\mathrm{NMR}$ $\left(\mathrm{CDCl}_{3}\right): \delta=125.7$ (d; C-3), 124.2 (d; C-2), 60.1 (d; C-7a), 55.0, 48.3 (2 d; C-3a, C-4), 50.0 (2 s; C-7, C-8), 29.2 (t; C-6), 23.4 (t; C-5), 20.3 (q; $\mathrm{CH}_{3}$ ), 18.3 (q; $\left.\mathrm{CH}_{3}\right), 15.5$ (q; $\mathrm{CH}_{3}$ ).

$$
\mathrm{C}_{12} \mathrm{H}_{18} \mathrm{~S} \text { (194.3) Ber. C } 74.16 \text { H } 9.34 \text { Gef. C } 74.29 \text { H } 9.39
$$

Acetalisierung von 1 mit Methanol: $40 \mathrm{mg}(0.19 \mathrm{mmol}) 1$ und $24 \mathrm{mg}(0.76 \mathrm{mmol})$ Methanol ergaben bei Raumtemp. und $p$-Toluolsulfonsäure-Katalyse $O$-MBT-Methanol (MBT-OMe, 11). $-{ }^{1} \mathrm{H}-\mathrm{NMR}\left(\mathrm{CDCl}_{3}\right): \delta=5.21(\mathrm{t}, J=3.5 \mathrm{~Hz} ; 1 \mathrm{H}, 2-\mathrm{H}), 3.84(\mathrm{~d}, J=11.5 \mathrm{~Hz} ; 1 \mathrm{H}, 7 \mathrm{a}-$ H), 3.32 (s; $\left.3 \mathrm{H}, \mathrm{OCH}_{3}\right), 3.3-2.9(\mathrm{~m} ; 1 \mathrm{H}, 3 \mathrm{a}-\mathrm{H}), 2.3-1.05$ (m; $7 \mathrm{H}$, Aliphaten-H), 1.04/0.98/ $0.78\left(3 \mathrm{~s} ; 9 \mathrm{H}, 3 \mathrm{CH}_{3}\right)$. Außerdem wurde ein endo-Anteil von $\approx 15 \%$ festgestellt.

Untersuchung der Acetalisierung von MBT-OH mit 1-Phenylethanol: $0.377 \mathrm{~g}(1.78 \mathrm{mmol})$ 1 und $0.643 \mathrm{~g}(5.27 \mathrm{mmol}) \mathrm{rac}-2$ wurden in $4 \mathrm{ml} \mathrm{CDCl}_{3}$ bei $0^{\circ} \mathrm{C}$ mit $10 \mathrm{mg} p$-Toluolsulfonsäure zur Katalyse versetzt. Im Verlauf von $48 \mathrm{~h}$ wurden zu bestimmten Zeiten je $100 \mu \mathrm{l}$ Reaktionsproben mit $2 \mu$ Triethylamin (zum Stoppen der Reaktion) vermischt und durch ${ }^{1}$ H-NMR-Spektroskopie das Diastereomerenverhältnis der $\alpha($ exo $)$-Anomeren sowie die relativen molaren Anteile der Schutzgruppe an 9 und $\beta($ endo)-Anomeren bestimmt. Die Ergebnisse sind in Tab. 3 dargestellt.

Tab. 3. Kinetische Untersuchung der Enantiomer-selektiven Acetalisierung von 1 mit überschüssigem rac-2

\begin{tabular}{|c|c|c|c|c|c|c|c|c|}
\hline Probe & $\begin{array}{l}\text { Zeitpunkt } \\
\text { der } \\
\text { Proben- } \\
\text { nahme }\end{array}$ & \multicolumn{5}{|c|}{$\begin{array}{l}\text { Relative molare Anteile } \\
\text { an Schutzgruppe } \\
\text { der entstehenden Reak- } \\
\text { tionsprodukte in \% }\end{array}$} & \multicolumn{2}{|c|}{$\begin{array}{l}\text { Relatives } \\
\text { Verhältnis } \\
\text { der } \alpha(\text { exo }) \\
\text { Anomeren }\end{array}$} \\
\hline $\begin{array}{l}1 \\
2 \\
3 \\
4 \\
5 \\
6 \\
7\end{array}$ & $\begin{array}{c}4.5 \mathrm{~min} \\
18.5 \mathrm{~min} \\
87 \mathrm{~min} \\
190 \mathrm{~min} \\
5.5 \mathrm{~h} \\
22 \mathrm{~h} \\
48 \mathrm{~h}\end{array}$ & 6 & $\begin{array}{r}4 \\
9 \\
24 \\
43 \\
49 \\
70 \\
74\end{array}$ & $\begin{array}{l}30 \\
37 \\
50 \\
45 \\
42 \\
18 \\
13\end{array}$ & $\begin{array}{r}59 \\
47 \\
18 \\
4\end{array}$ & $\begin{array}{r}7 \\
8 \\
8 \\
9 \\
12 \\
13\end{array}$ & $\begin{array}{l}1 \\
1 \\
1 \\
1 \\
1.2 \\
3.9 \\
5.7\end{array}$ & $\begin{array}{l}7.3 \\
4 \\
2.1 \\
1 \\
1 \\
1 \\
1\end{array}$ \\
\hline
\end{tabular}

a) Der molare Prozentanteil an 9 ist nur halb so groß, da ein Molekül 9 als Dimeres zwei Åquivalenten Schutzgruppe entspricht. Die Diskussion der experimentellen Daten findet sich im allgemeinen Teil.

Thermodynamisch gelenkte Acetalisierung mit rac-2: Im davor angeführten Acetalisierungs/ Äquilibrierungsversuch wurden nach $22 \mathrm{~h} 3 \mathrm{ml}$ Reaktionslösung (284 mg (1.34 mmol) Edukt 1) bei $0^{\circ} \mathrm{C}$ mit $30 \mu l$ Triethylamin versetzt, festes Natriumhydrogencarbonat und Natriumsulfat zugegeben, filtriert und i. Vak. eingedampft. Das erhaltene Produktgemisch (3:4 = 79:21 laut NMR) wurde durch SC (65 g Triethylamin-imprägniertes Kieselgel feinst; Eluens: $\mathrm{PE} / \mathrm{E}=150: 1)$ aufgetrennt in $35 \mathrm{mg} 12\left(\left(S^{*}\right)-\beta(\right.$ endo)-Anomeres), $22 \mathrm{mg}$ Produktgemisch (Hauptmenge dem $\left(R^{*}\right)$ - $\beta($ endo)-Anomeren 13 zugeordnet), $237 \mathrm{mg} 3$ und $77 \mathrm{mg}$ einer Fraktion aus $15 \% 3$ und $85 \%$ 4. Gesamtausb. $370 \mathrm{mg}(88 \%)$, davon $249 \mathrm{mg} \mathrm{(59 \% )} \mathrm{3,} 65 \mathrm{mg}$ $(15 \%) 4,34 \mathrm{mg}(8 \%) 12$ und $22 \mathrm{mg}(5 \%)$ einer Fraktion, welche hauptsächlich aus 13 bestand.

Kinetisch gelenkte Acetalisierung mit rac-2: $225 \mathrm{mg}(1.06 \mathrm{mmol}) 1$ und $390 \mathrm{mg}(3.18 \mathrm{mmol})$ rac-2 wurden in $4 \mathrm{ml} \mathrm{CDCl}{ }_{3}$ bei $0^{\circ} \mathrm{C}$ mit $10 \mathrm{mg} p$-Toluolsulfonsäure versetzt und (nach 
Umsatzkontrolle durch ${ }^{1} \mathrm{H}-\mathrm{NMR}$-Spektrum) nach $11 \mathrm{~min} 30 \mu \mathrm{l}$ Triethylamin zugegeben. Es wurde festes Natriumhydrogencarbonat und Natriumsulfat zugegeben, filtriert und i. Vak. eingedampft. Das Produktgemisch (9:3:4 $\approx 25: 19: 56$ mol-\% laut ${ }^{1} \mathrm{H}-\mathrm{NMR}$, das sind 9:3:4 $\approx 40: 15: 45 \% \mathrm{MBT}$ ) wurde durch SC analog getrennt. Eluens: erst PE: $\mathrm{E}=150: 1$, dann (für 9) 25:1. Es wurden $38 \mathrm{mg} \mathrm{3,15} \mathrm{mg}$ Gemisch (27\% 3, 73\% 4), $114 \mathrm{mg} 4$ und $75 \mathrm{mg}$

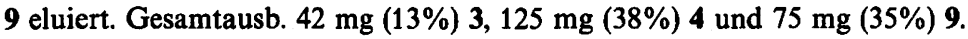

(R)-O-MBT-1-Phenylethanol ( $\left[2 S-\left[2 \alpha\left(S^{*}\right), 3 a \alpha, 4 \alpha, 7 \alpha, 7 a \alpha\right]\right]$-Octahydro-7,8,8-trimethyl-2(1-phenylethoxy)-4,7-methanobenzo[b]thiophen) (3): Farblose Kristalle, Schmp. $98-99^{\circ} \mathrm{C}$ (aus PE). $-[\alpha]_{\mathbb{D}}^{20}=+311^{\circ}(c=2.20$ in Tetrachlormethan). - IR (KBr): $2940(\mathrm{CH})$, 1090, 1045, $1020 \mathrm{~cm}^{-1}(\mathrm{C}-\mathrm{O})$. $-{ }^{1} \mathrm{H}-\mathrm{NMR}\left(\mathrm{CDCl}_{3}\right): \delta=7.30$ (s; 5H, Aromaten-H), 5.11 (t, $J=3.5 \mathrm{~Hz}$; $1 \mathrm{H}, 2-\mathrm{H}$ ), 4.71 (q, $J=6.5 \mathrm{~Hz} ; 1 \mathrm{H}, \mathrm{PhCH}$ ), 3.98/3.95/3.86/3.83 (dd; $1 \mathrm{H}, 7 \mathrm{a}-$ H), $3.38-2.92(\mathrm{~m} ; 1 \mathrm{H}, 3 \mathrm{a}-\mathrm{H}), 2.09-1.06(\mathrm{~m} ; 10 \mathrm{H}$, Aliphaten-H, darin: $1.42, \mathrm{~d}, J=6.5 \mathrm{~Hz}$; $\left.\mathrm{PhCCH}_{3}\right), 1.05 / 0.96 / 0.78\left(3 \mathrm{~s} ; 9 \mathrm{H}, 3 \mathrm{CH}_{3}\right) .-{ }^{13} \mathrm{C}-\mathrm{NMR}\left(\mathrm{CDCl}_{3}\right): \mathrm{MBT}: \delta=92.4$ (d; C-2), 60.7 (d; C-7a), 52.9 (s; C-7), 49.0 (s; C-8), 47.7 (d; C-4), 45.4 (d; C-3a), 38.4 (t; C-3), 28.8 (t; C-6), 21.4 (q; $\left.\mathrm{CH}_{3}\right), 20.9$ (t; C-5), 18.5 (q; $\left.\mathrm{CH}_{3}\right), 15.9$ (q; $\left.\mathrm{CH}_{3}\right)$; Alkoxyrest: 143.0 (s; $\left.\mathrm{C}(\mathrm{Ph})-1\right)$, 128.4 (d; C(Ph)-3, C(Ph)-5), 127.4 (d; C(Ph)-4), 126.6 (d; C(Ph)-2, C(Ph)-6, 75.5 (d; CHPh), $24.2\left(\mathrm{q} ; \mathrm{PhC}-\mathrm{CH}_{3}\right)$.

Abspaltung der Schutzgruppe: $\mathrm{Zu}$ einer Lösung von 3 in Chloroform wurde etwas pToluolsulfonsäure gegeben und bei Raumtemp. $1 \mathrm{~h}$ stehengelassen. Es wurden festes Natriumhydrogencarbonat und Natriumsulfat zugegeben, filtriert und eingedampft. Die Abspaltung wurde durch NMR kontrolliert. Durch SC (Triethylamin-imprägniertes Kieselgel) wurde der Alkohol $(R)-2$ von 10 abgetrennt. $[\alpha]_{\mathrm{D}}^{20}=+48^{\circ}$ (Dichlormethan).

(S)-O-MBT-1-Phenylethanol ( $\left[2 S-\left[2 \alpha\left(R^{*}\right), 3 a \alpha, 4 \alpha, 7 \alpha, 7 a \alpha\right]\right]-O c t a h y d r o-7,8,8$-trimethyl-2(1-phenylethoxy)-4,7-methanobenzo[b]thiophen (4): Farbloses Öl, Sdp. $100^{\circ} \mathrm{C} / 0.02$ Torr (Luftbad); $[\alpha]_{\mathrm{D}}^{20}=+220^{\circ}(c=1.08$ in Tetrachlormethan). - IR (KBr): $2935(\mathrm{CH}), 1080 /$ $1060 / 1040 \mathrm{~cm}^{-1}(\mathrm{C}-\mathrm{O}) .-{ }^{1} \mathrm{H}-\mathrm{NMR}\left(\mathrm{CDCl}_{3}\right): \delta=7.31(\mathrm{~s} ; 5 \mathrm{H}$, Aromaten-H), $5.51(\mathrm{t}, J=$ $3.8 \mathrm{~Hz} ; 1 \mathrm{H}, 2-\mathrm{H}), 4.70$ (q, $J=6.5 \mathrm{~Hz} ; 1 \mathrm{H}, \mathrm{PhCH}), 3.79$ (d, $J=10.5 \mathrm{~Hz} ; 1 \mathrm{H}, 7 \mathrm{a}-\mathrm{H})$, $3.30-2.84(\mathrm{~m} ; 1 \mathrm{H}, 3 \mathrm{a}-\mathrm{H}), 2.16-1.0(\mathrm{~m} ; 10 \mathrm{H}$, Aliphaten-H, darin: $1.44, \mathrm{~d}, J=6.5 \mathrm{~Hz}$; $\left.\mathrm{PhCCH}_{3}\right), 1.00 / 0.95 / 0.72\left(3 \mathrm{~s} ; 9 \mathrm{H}, 3 \mathrm{CH}_{3}\right) .-{ }^{13} \mathrm{C}-\mathrm{NMR}\left(\mathrm{CDCl}_{3}\right)$ : MBT: $\delta=92.4(\mathrm{~d} ; \mathrm{C}-2)$, 61.0 (d; C-7a), 52.6 (s; C-7), 49.2 (s; C-8), 48.0 (d; C-4), 45.1 (d; C-3a), 38.6 (t; C-3), 28.9 (t; C-6), 21.3 (q; $\left.\mathrm{CH}_{3}\right), 21.2$ (t; C-5), 18.6 (q; $\left.\mathrm{CH}_{3}\right), 15.9$ (q, $\left.\mathrm{CH}_{3}\right)$; Alkoxyrest: 143.8 (s; C(Ph)-1), 128.2 (d; C(Ph)-3, C(Ph)-5), 127.2 (d; C(Ph)-4), 126.1 (d; C(Ph)-2, C(Ph)-6), 75.4 (d; CHPh), 21.6 (q; $\mathrm{PhC}-\mathrm{CH}_{3}$ ).

$$
\mathrm{C}_{20} \mathrm{H}_{28} \mathrm{OS}(316.5) \text { Ber. C } 75.90 \text { H } 8.92
$$

3: Gef. C 75.77 H 9.00 4: Gef. C 75.93 H 8.82

Abspaltung der Schutzgruppe: Eine Lösung von $50 \mathrm{mg} 4$ in Chloroform wurde $2 \mathrm{~h}$ mit etwas $p$-Toluolsulfonsäure stehengelassen. Isolierung wie für $(R)-2$ beschrieben ergab $13 \mathrm{mg}$ $(68 \%)(S)-2 .-[\alpha]_{\mathrm{D}}^{20}=-41^{\circ}$ (Dichlormethan).

[2R- $\left.\left[2 \alpha\left(S^{*}\right), 3 a \beta, 4 \beta, 7 \beta, 7 a \beta\right]\right]-O c t a h y d r o-7,8,8-t r i m e t h y l-2-(1-p h e n y l e t h o x y)-4,7-m e t h a n o-$ benzo[b]thiophen (12): Farbloses Öl, Sdp. $100^{\circ} \mathrm{C} / 0.02$ Torr (Luftbad). $-[\alpha]_{\mathrm{D}}^{20}=-84^{\circ}(c=$ 0.60/Dichlormethan). - IR (KBr): 2920 (CH), 1080/1050/1025 (C-O). - ${ }^{1} \mathrm{H}-\mathrm{NMR}$ $\left(\mathrm{CDCl}_{3}\right): \delta=7.30(\mathrm{~s} ; 5 \mathrm{H}$, Aromaten-H), 5.20/5.17/5.13/5.10 (dd; $1 \mathrm{H}, 2-\mathrm{H}), 4.71(\mathrm{q}, J=$ $6.5 \mathrm{~Hz} ; 1 \mathrm{H}, \mathrm{PhCH}), 3.79$ (d, $J=12 \mathrm{~Hz} ; 1 \mathrm{H}, 7 \mathrm{a}-\mathrm{H}), 3.26-2.84$ (m; $1 \mathrm{H}, 3 \mathrm{a}-\mathrm{H}), 2.38-1.0$ (m; $10 \mathrm{H}$, Aliphaten-H, darin: $\left.1.45, \mathrm{~d}, J=6.5 \mathrm{~Hz} ; \mathrm{PhCCH}_{3}\right), 0.99\left(\mathrm{~s} ; 6 \mathrm{H}, 2 \mathrm{CH}_{3}\right), 0.77(\mathrm{~s} ; 3 \mathrm{H}$, $\left.\mathrm{CH}_{3}\right) .-{ }^{13} \mathrm{C}-\mathrm{NMR}\left(\mathrm{CDCl}_{3}\right)$ : MBT: $\delta=91.8(\mathrm{~d} ; \mathrm{C}-2), 63.6$ (d; C-7a), 52.2 (s; C-7), 49.6 (s; C-8), 49.4 (d; C-3a), 48.8 (d; C-4), 39.3 (t; C-3), 29.6 (t; C-6), 21.2 (t, q; C-5, CH$_{3}$ ), 18.7 (q; $\mathrm{CH}_{3}$ ), 16.0 (q; $\mathrm{CH}_{3}$ ); Alkoxyrest: 142.9 (s, C(Ph)-1), 128.5 (d; $\left.\mathrm{C}(\mathrm{Ph})-3, \mathrm{C}(\mathrm{Ph})-5\right), 127.5$ (d; $\mathrm{C}(\mathrm{Ph})-4), 126.8$ (d; C(Ph)-2, C(Ph)-6), 76.0 (d; $\mathrm{CHPh}$ ), 24.1 (q; $\mathrm{PhCCH}_{3}$ ).

Chem. Ber. $118(1985)$ 
Folgende Signallagen des ${ }^{1} \mathrm{H}$-NMR-Spektrums einer SC-Fraktion der thermodynamisch gelenkten Acetalisierung wurden dem $\left(R^{*}\right)-\beta(e n d o)$-Anomeren $\left(\left[2 R-\left[2 \alpha\left(R^{*}\right), 3 \alpha \beta\right.\right.\right.$,$4 \beta, 7 \beta, 7 a \beta]$ ]-Octahydro-7,8,8-trimethyl-2-(1-phenylethoxy)-4,7-methanobenzo[b]thiophen) (13) zugeordnet: ${ }^{1} \mathrm{H}-\mathrm{NMR}\left(\mathrm{CDCl}_{3}\right): \delta=7.3$ (s; 5H, Aromaten-H), 5.61/5.58/5.53/5.50 (dd; $1 \mathrm{H}, 2-\mathrm{H}), 4.7$ (q, $J=6.5 \mathrm{~Hz} ; 1 \mathrm{H}, \mathrm{PhCH}), 3.75(\mathrm{~d}, J=11 \mathrm{~Hz} ; 1 \mathrm{H}, 7 \mathrm{a}-\mathrm{H}), 3.3-2.9(\mathrm{~m} ; 1 \mathrm{H}$, 3a-H), 2.5-1.0 (m; 10H, Aliphaten-H), 0.97 (s; 6H, $\left.2 \mathrm{CH}_{3}\right), 0.77\left(\mathrm{~s} ; 3 \mathrm{H}, \mathrm{CH}_{3}\right.$ ).

1) Chirale Lactole, II. Mitteil.: C. R. Noe, Chem. Ber. 115, 1591 (1982); zum Begriff „Enantiomer-Selektivität" vgl. auch Y. Okamoto, K. Suzuki, T. Kitayama, H. Yuki, H. Kageyama, K. Miki, N. Tanaka, N. Kasai, J. Am. Chem. Soc. 104, 17, 4618 (1982).

2) C. R. Noe, M. Knollmüller, E. Wagner und H. Völlenkle, Chem. Ber. 118, 1733 (1985).

3) 3a) R. U. Lemieux und S. Koto, Tetrahedron 30, $1933(1974)$. - ${ }^{3 b)} R$. U. Lemieux, S. Koto und D. Voisin, Am. Chem. Soc., Symposium Ser. 87, 17 (1979).

4) Der Van der Waals-Radius beträgt für ein Sauerstoffatom 140, für ein Schwefelatom $185 \mathrm{pm}$.

5) J. W. Frost, Aldrichimica Acta 16, (2), 39 (1983).

6) B. S. Pedersen, S. Scheibye, N. H. Nilsson und S.-O. Lawesson, Bull. Soc. Chim. Belg. 87, 223 (1978).

7) I. Mitteil:: C. R. Noe, Chem. Ber. 115, 1576 (1982).

8) A. J. Kirby, The Anomeric Effect and Related Stereoelectronic Effects at Oxygen, Springer-Verlag 1983.

9) Bei Raumtemperatur bestand eine große Neigung zu Bildung der Anhydroform 10.

10) Dabei zeigten NMR-Versuche, daß das thermodynamisch stabilere $\left(R^{*}\right)$-Diastereomere 3 wesentlich langsamer spaltete als das $\left(S^{*}\right)$-Diastereomere 4.

11) Die entsprechenden Werte für die Protonen an C-2 und an C-7a betrugen bei Modell I jeweils $\Delta \delta=.0 .37 \mathrm{ppm}$.

12) P. Main, MULTAN 78, University of York, England (1978).

13) G. Sheldrick: SHELX 76. Program for Crystall Structure Determination. - Univ. Cambridge, England (1976).

14) Der hier angegebene Torsionswinkel wird als $\mathrm{C} 12-\mathrm{O}-\mathrm{C} 2-$ S-Diederwinkel gemessen; er entspricht einem $\Phi^{\mathrm{H}}$-Winkel (Torsionswinkel $\mathrm{C} 12-\mathrm{O}-\mathrm{C} 2-\mathrm{H}(\mathrm{C} 2)$ ) von $38.2^{\circ}$ bzw. $45.7^{\circ}$ bei der analogen Sauerstoffverbindung. Vgl. Lit.: $R$. U. Lemieux und $S$. Koto, Tetrahedron 30, 1933 (1974). Die entsprechenden $\Phi^{H}$-Werte von Methyl- $\beta$ - und - $\alpha$-D-glucopyranosiden mit $48^{\circ}$ und $57^{\circ}$ wurden durch Röntgenstrukturanalyse bestimmt.

15) Das Ausmaß der Enantiomer-Selektivität kann nicht mit Sicherheit allein auf die unterschiedliche Größe des Schwefels gegenüber dem Sauerstoff zurückgeführt werden, sondern es müssen auch andere Effekte, wie die unterschiedliche Geometrie der Acetalsysteme (z. B. stärkere Knickung des Thiolactolringes) oder elektronische Effekte in Betracht gezogen werden.

16) Der Rest bestand zum großen Teil aus 12 und 13; er wurde abgeschätzt durch die quantitative säulenchromatographische Isolierung bei kinetischer und thermodynamischer Acetalisierung.

$[296 / 84]$ 